\title{
6-Deoxy- and 11-hydroxy-tolypodiols: Meroterpenoids from the Cyanobacterium HT-58-2
}

Joshua R. Gurr ${ }^{\dagger}$, Timothy J. O’Donnell, ${ }^{\dagger}$ Yuheng Luo ${ }^{\dagger}$, Wesley Y. Yoshida ${ }^{\dagger}$, Mary L. Hall', Alejandro M.S. Mayer ${ }^{\ddagger}$, Rui Sun ${ }^{\dagger}$, and Philip G. Williams ${ }^{\dagger^{*}}$

\section{Affiliation}

† Department of Chemistry, 2545 McCarthy Mall, Honolulu, HI 96822

‡ Department of Pharmacology, Chicago College of Osteopathic Medicine, Midwestern University, $55531^{\text {st }}$ Street, Downers Grove, IL 60515

\section{Corresponding Author}

Prof. Dr. Philip Williams, Department of Chemistry, University of Hawaii at Manoa, Honolulu, Hawaii, USA, 96822. E-mail: philipwi@hawaii.edu Phone: 8089565720 Fax: 8089565908 


\section{Table of Contents}

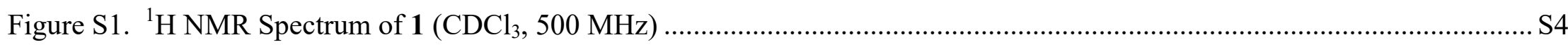

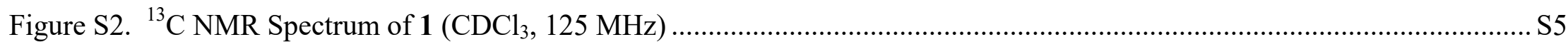

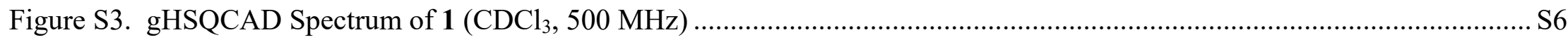

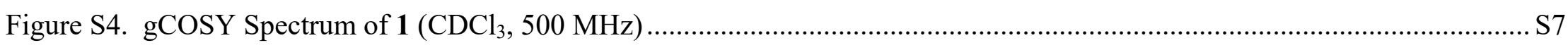

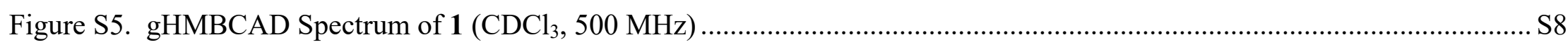

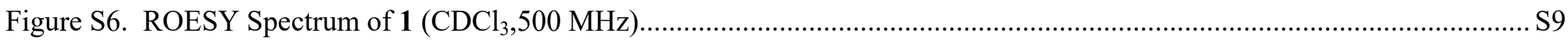

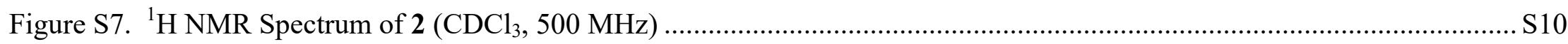

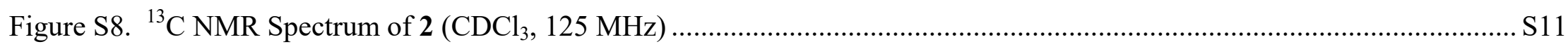

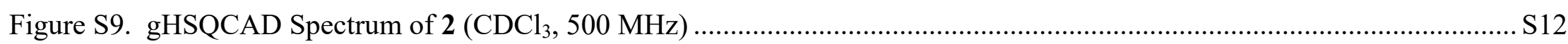

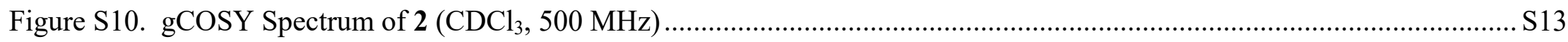

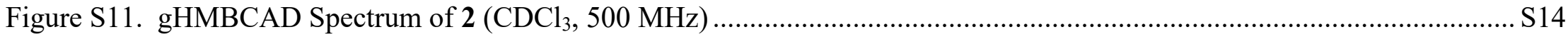

Figure S12. 1D-TOCSY Spectrum of $\mathbf{2}\left(\mathrm{CDCl}_{3}, 500 \mathrm{MHz}\right)$ irradiating resonance at $2.85 \mathrm{ppm}$.................................................... S15

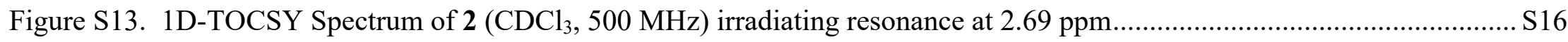

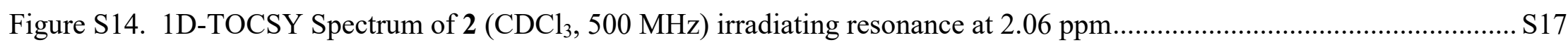

Table S1. Optimized Conformers of $\mathbf{1}$ - Energy and Abundance..................................................................................... S18

Table S2. Cartesian Coordinates For The Re-Optimized Conformer 1 Of Compound 1 …….................................................... S21

Table S3. Cartesian Coordinates For The Re-Optimized Conformer 2 Of Compound 1 1........................................................... S21

Table S4. Cartesian Coordinates For The Re-Optimized Conformer 3 Of Compound 1 1.......................................................... S22

Table S5. Cartesian Coordinates For The Re-Optimized Conformer 4 Of Compound 1 1............................................................... S23

Table S6. Cartesian Coordinates For The Re-Optimized Conformer 5 Of Compound 1 1............................................................. S24 
Table S7. Cartesian Coordinates For The Re-Optimized Conformer 6 Of Compound 1

Table S8. Cartesian Coordinates For The Re-Optimized Conformer 7 Of Compound 1

Table S9. Cartesian Coordinates For The Re-Optimized Conformer 8 Of Compound 1

Table S10. Cartesian Coordinates For The Re-Optimized Conformer 9 Of Compound 1 S28

Table S11. Cartesian Coordinates For The Re-Optimized Conformer 10 Of Compound 1. S29

Figure S15. Comparison of Calculated ECD spectra for $\mathbf{1}$ and similarity factors. $\mathrm{S} 30$ 
Figure S1. ${ }^{1} \mathrm{H}$ NMR Spectrum of $1\left(\mathrm{CDCl}_{3}, 500 \mathrm{MHz}\right)$

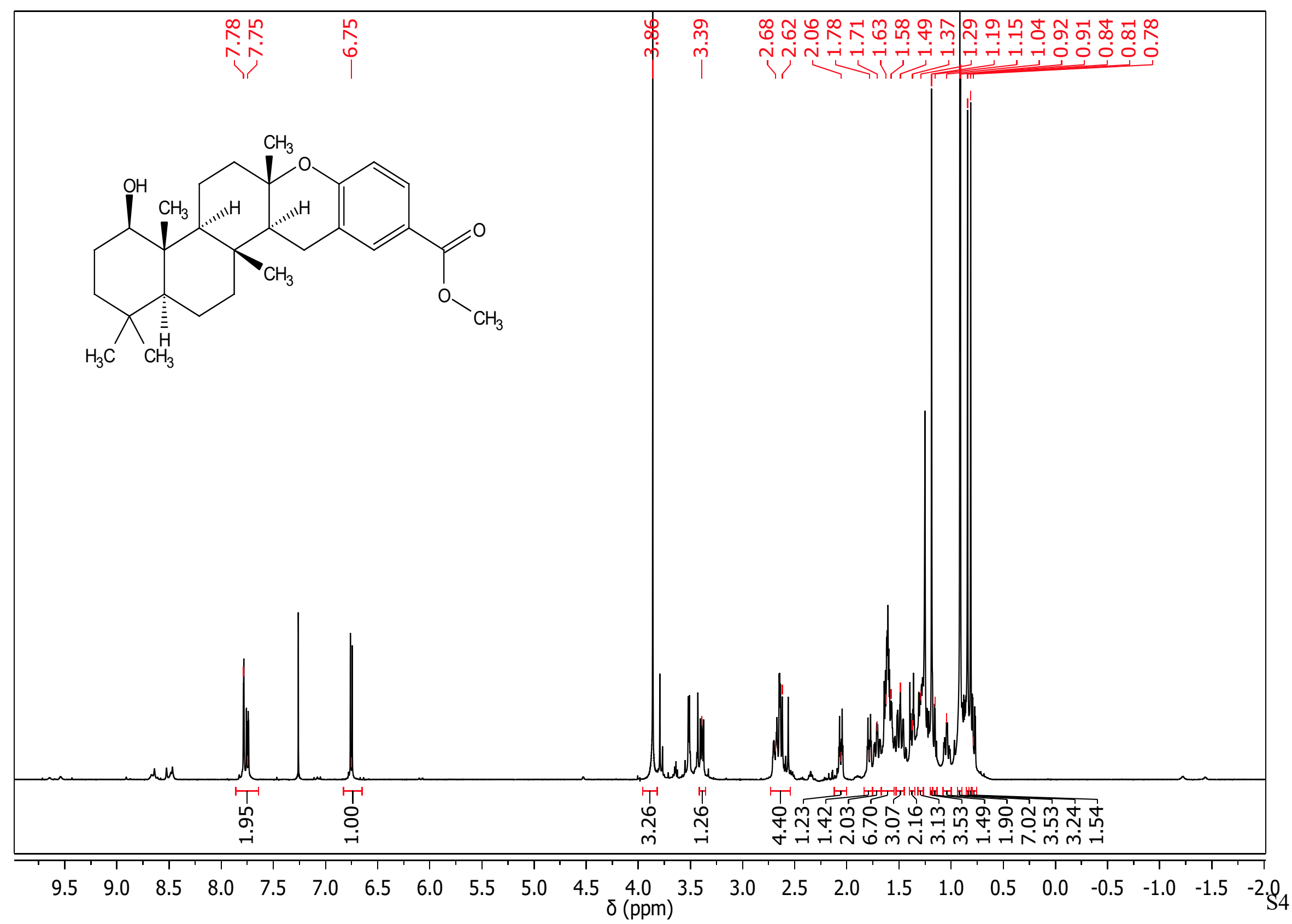


Figure S2. ${ }^{13} \mathrm{C}$ NMR Spectrum of $1\left(\mathrm{CDCl}_{3}, 125 \mathrm{MHz}\right)$

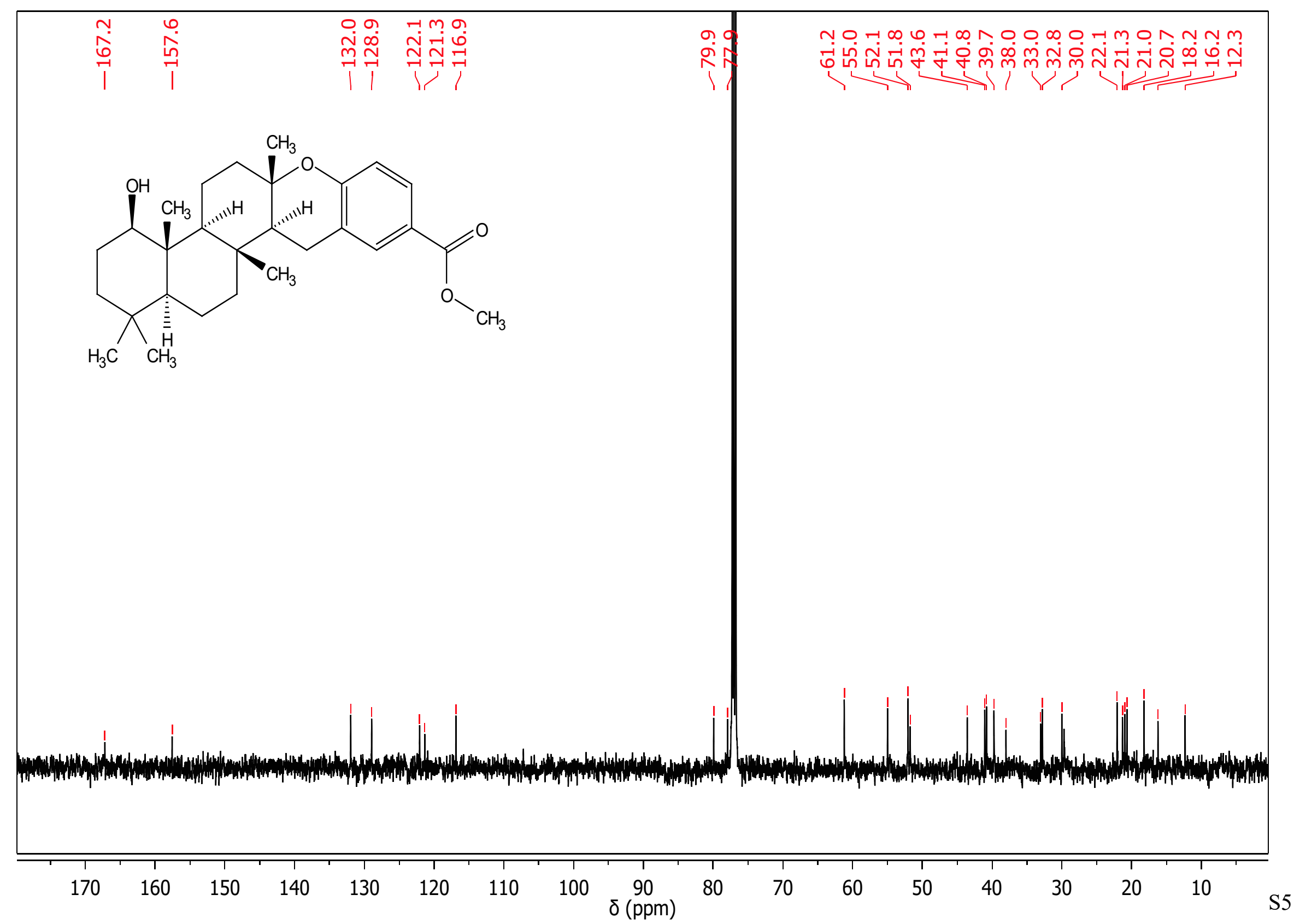


Figure S3. gHSQCAD Spectrum of $1\left(\mathrm{CDCl}_{3}, 500 \mathrm{MHz}\right)$

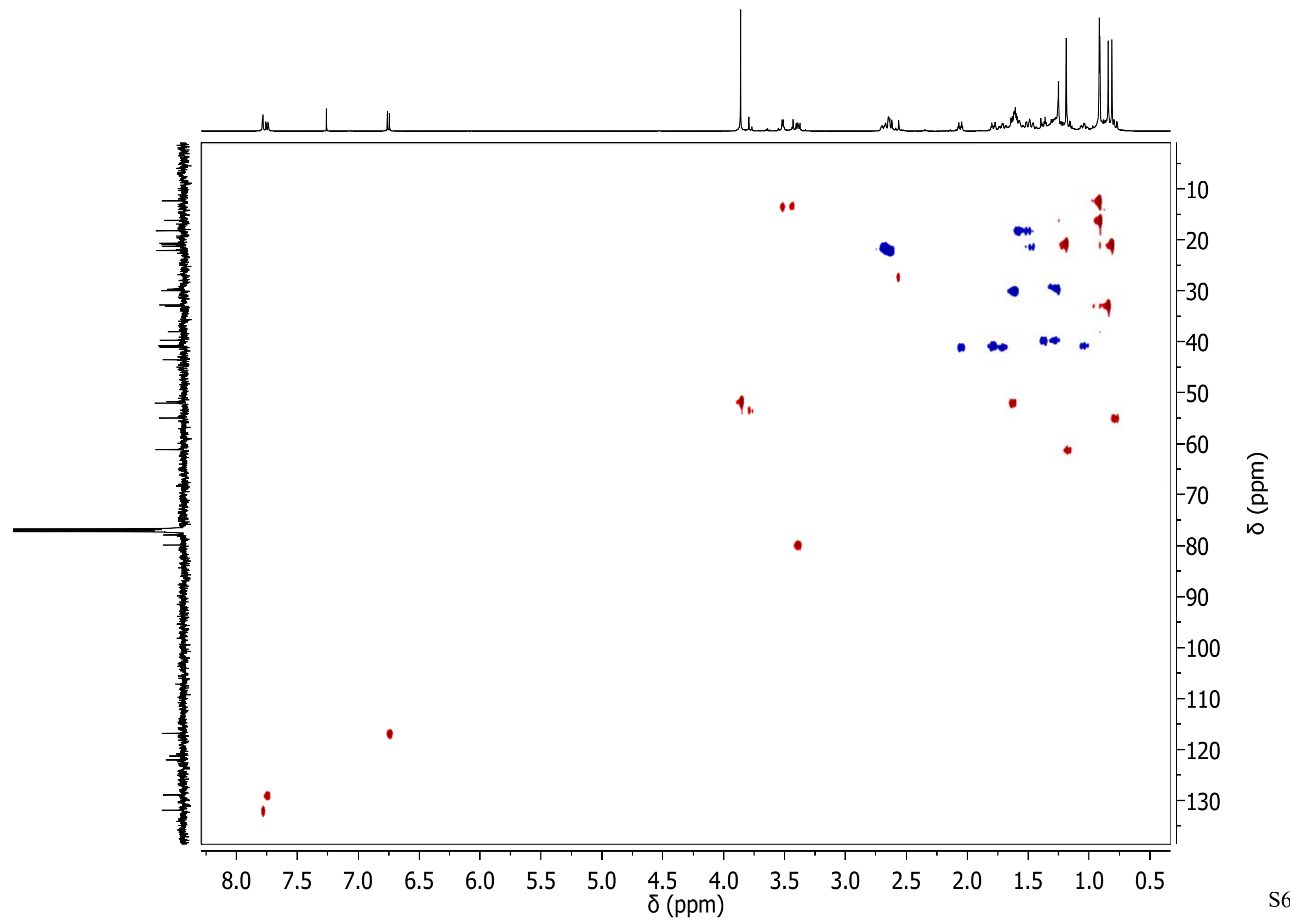


Figure S4. gCOSY Spectrum of $1\left(\mathrm{CDCl}_{3}, 500 \mathrm{MHz}\right)$

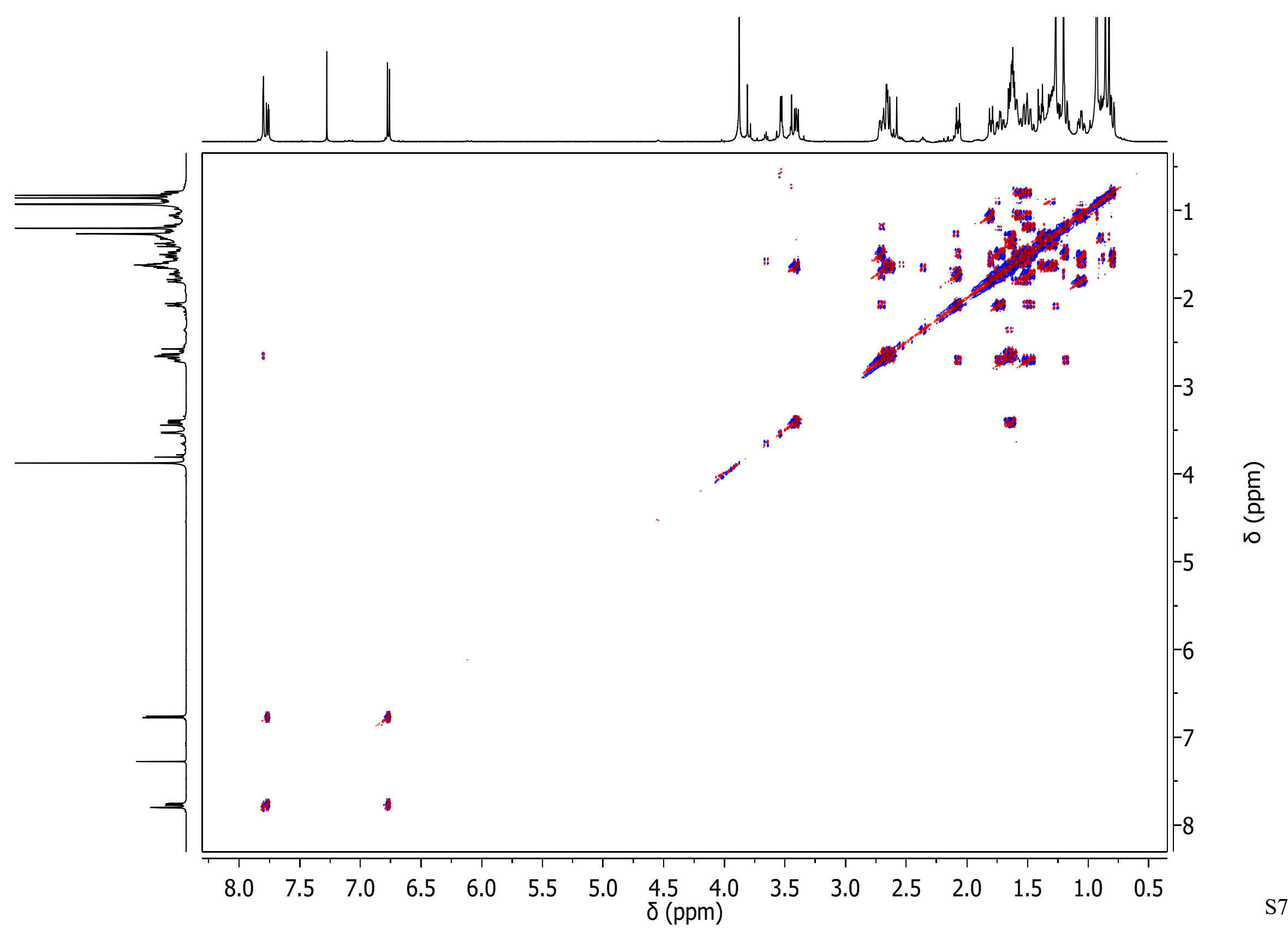


Figure S5. gHMBCAD Spectrum of $1\left(\mathrm{CDCl}_{3}, 500 \mathrm{MHz}\right)$

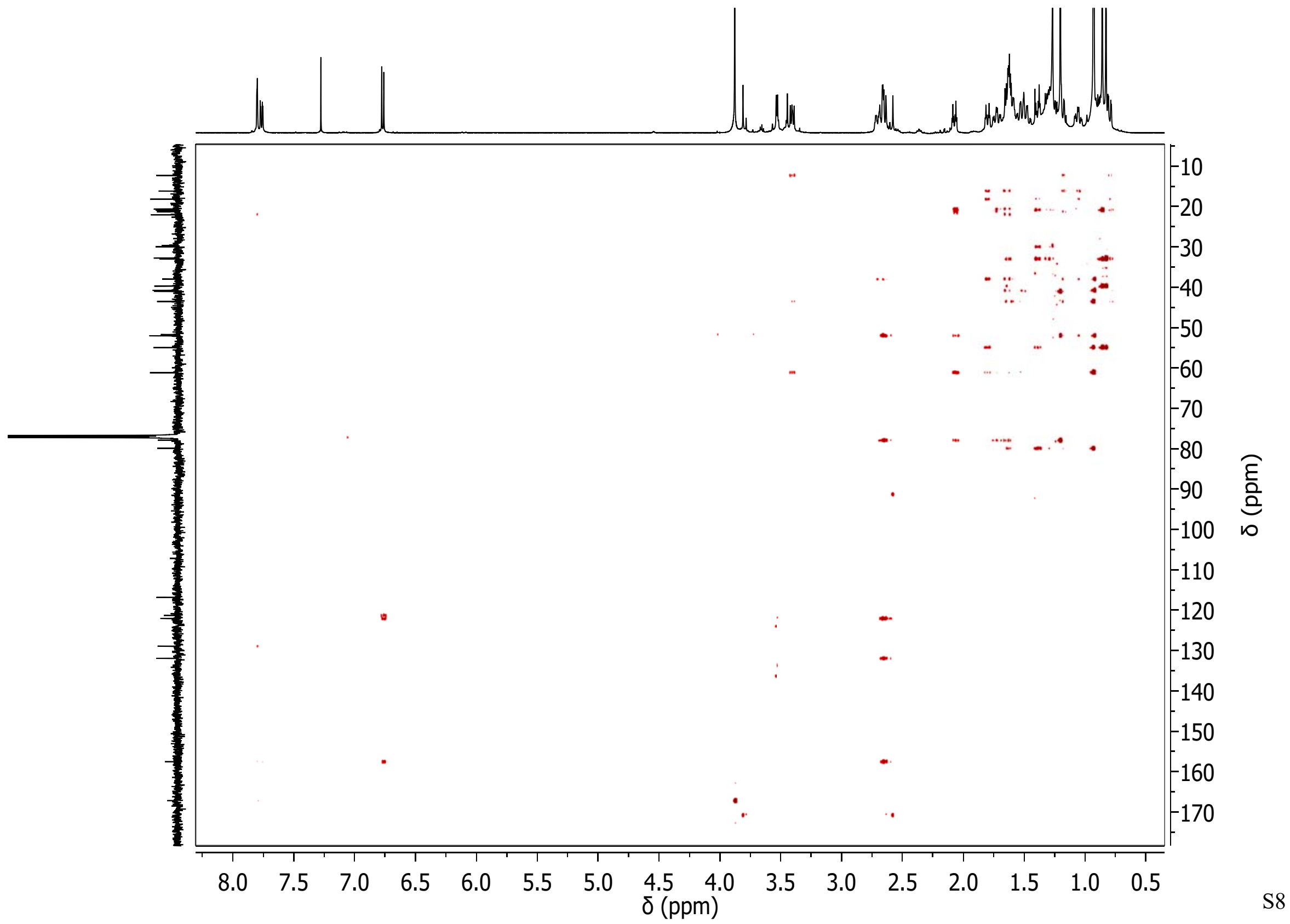


Figure S6. ROESY Spectrum of $1\left(\mathrm{CDCl}_{3}, 500 \mathrm{MHz}\right)$

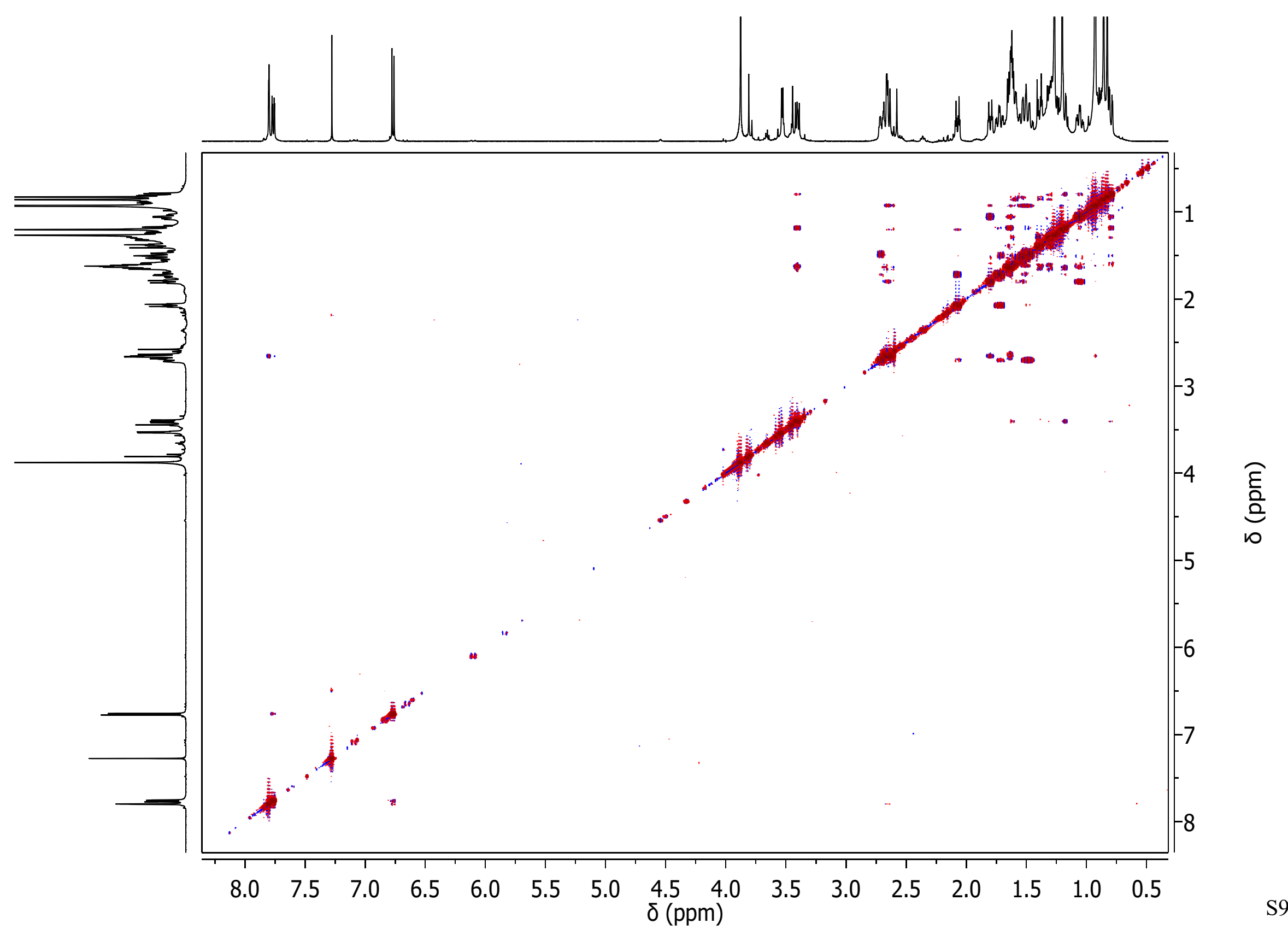


Figure S7. ${ }^{1} \mathrm{H}$ NMR Spectrum of $2\left(\mathrm{CDCl}_{3}, 500 \mathrm{MHz}\right)$

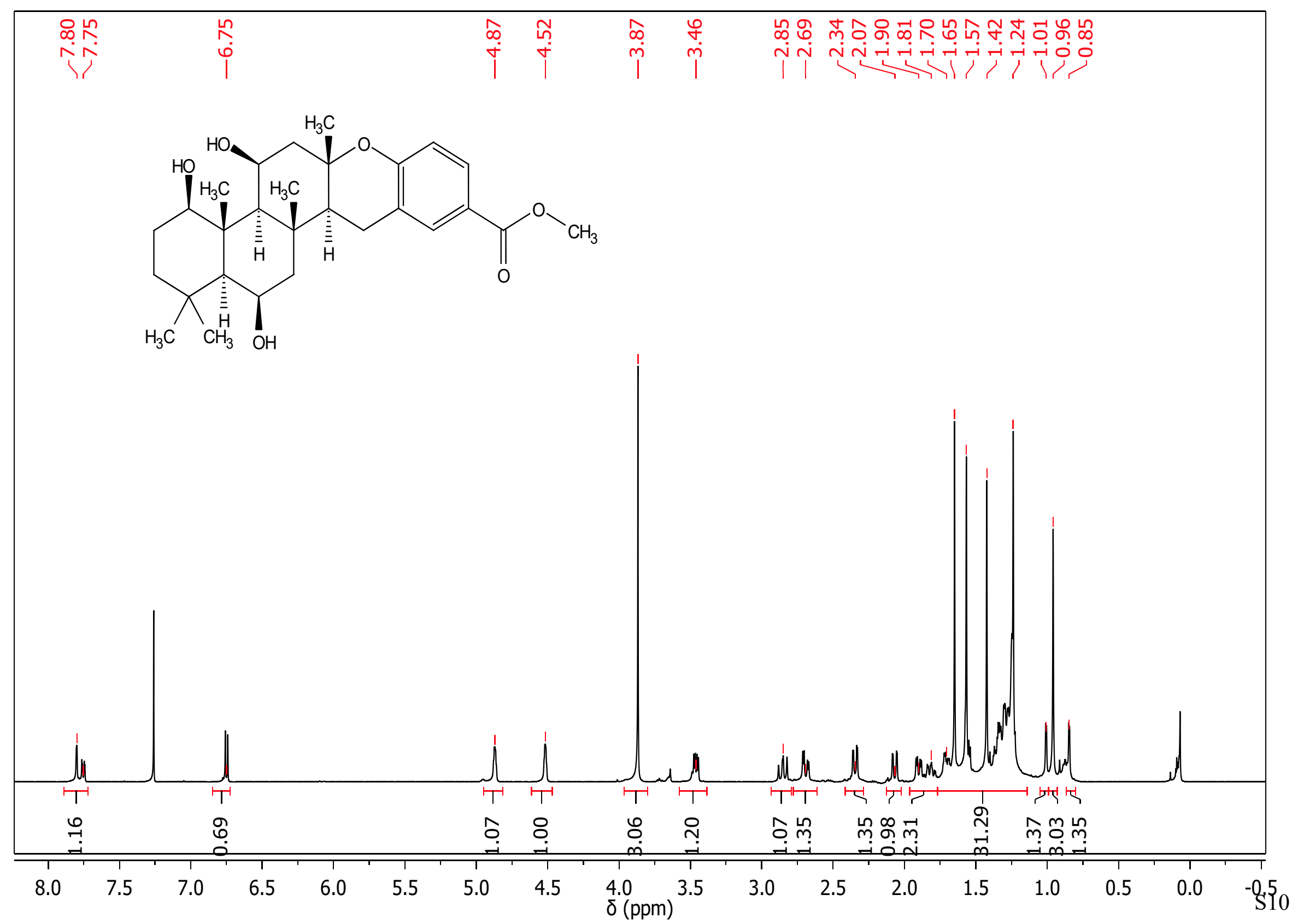


Figure S8. ${ }^{13} \mathrm{C}$ NMR Spectrum of $2\left(\mathrm{CDCl}_{3}, 125 \mathrm{MHz}\right)$

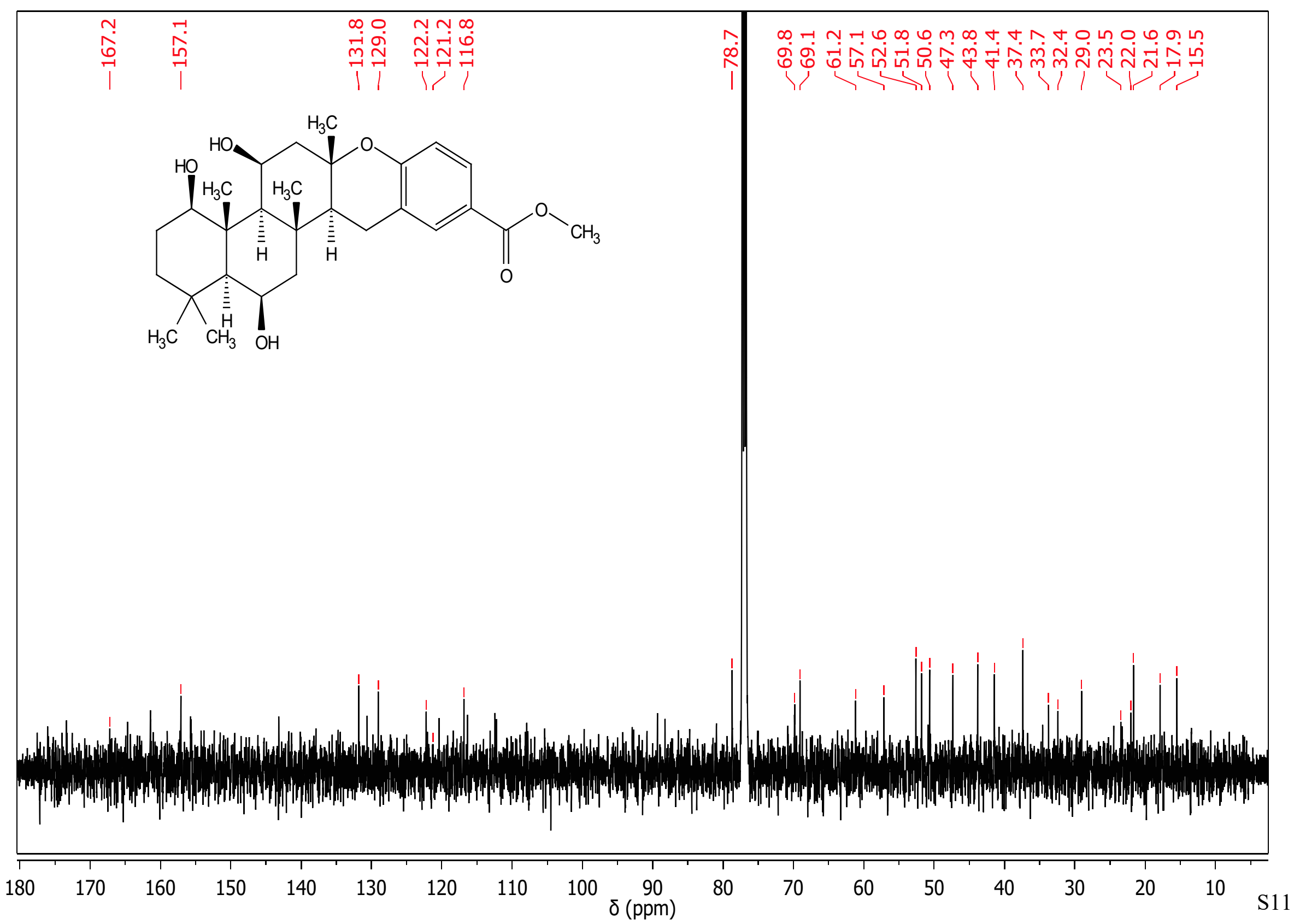


Figure S9. gHSQCAD Spectrum of $2\left(\mathrm{CDCl}_{3}, 500 \mathrm{MHz}\right)$

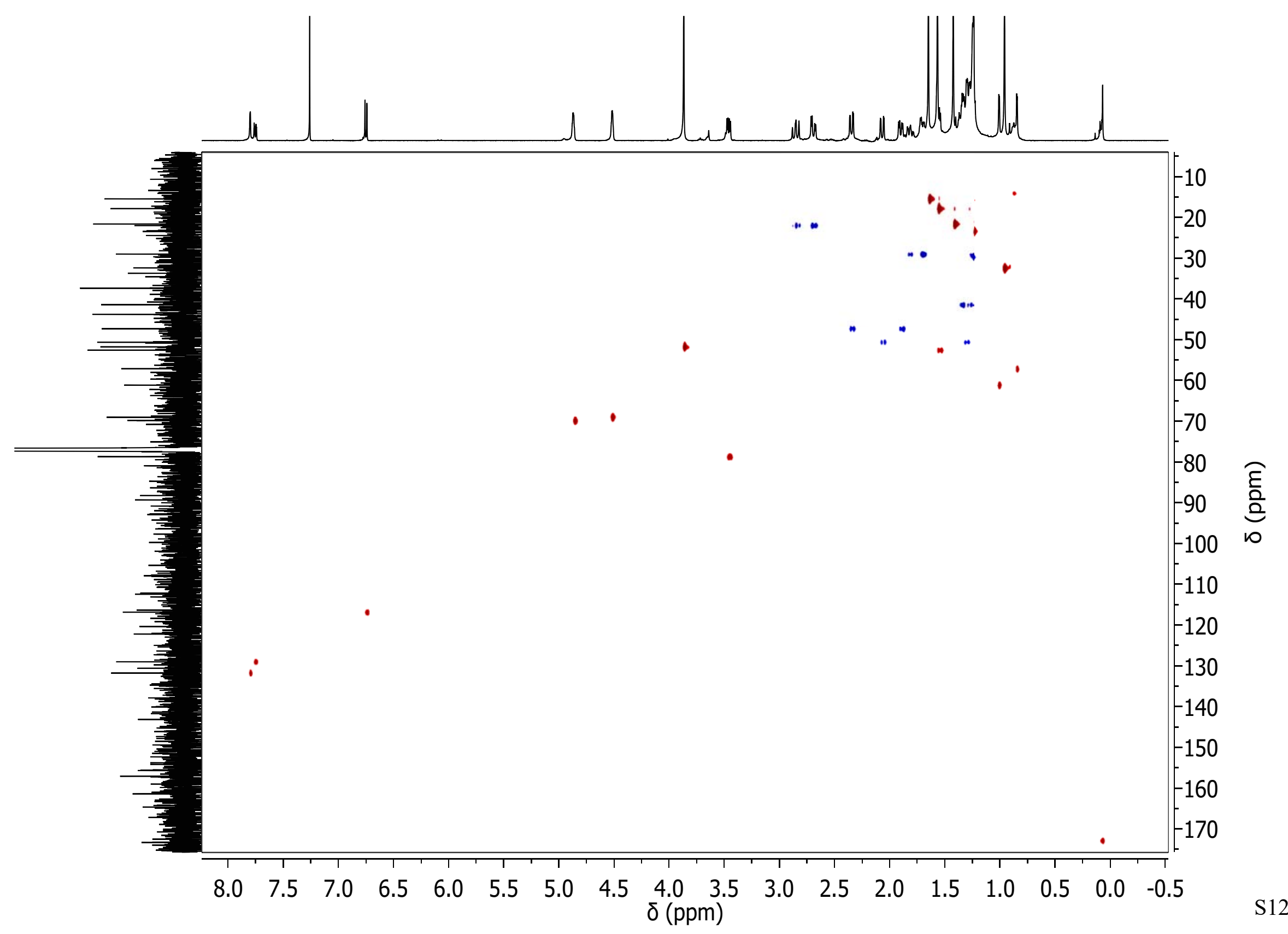


Figure S10. gCOSY Spectrum of $2\left(\mathrm{CDCl}_{3}, 500 \mathrm{MHz}\right)$

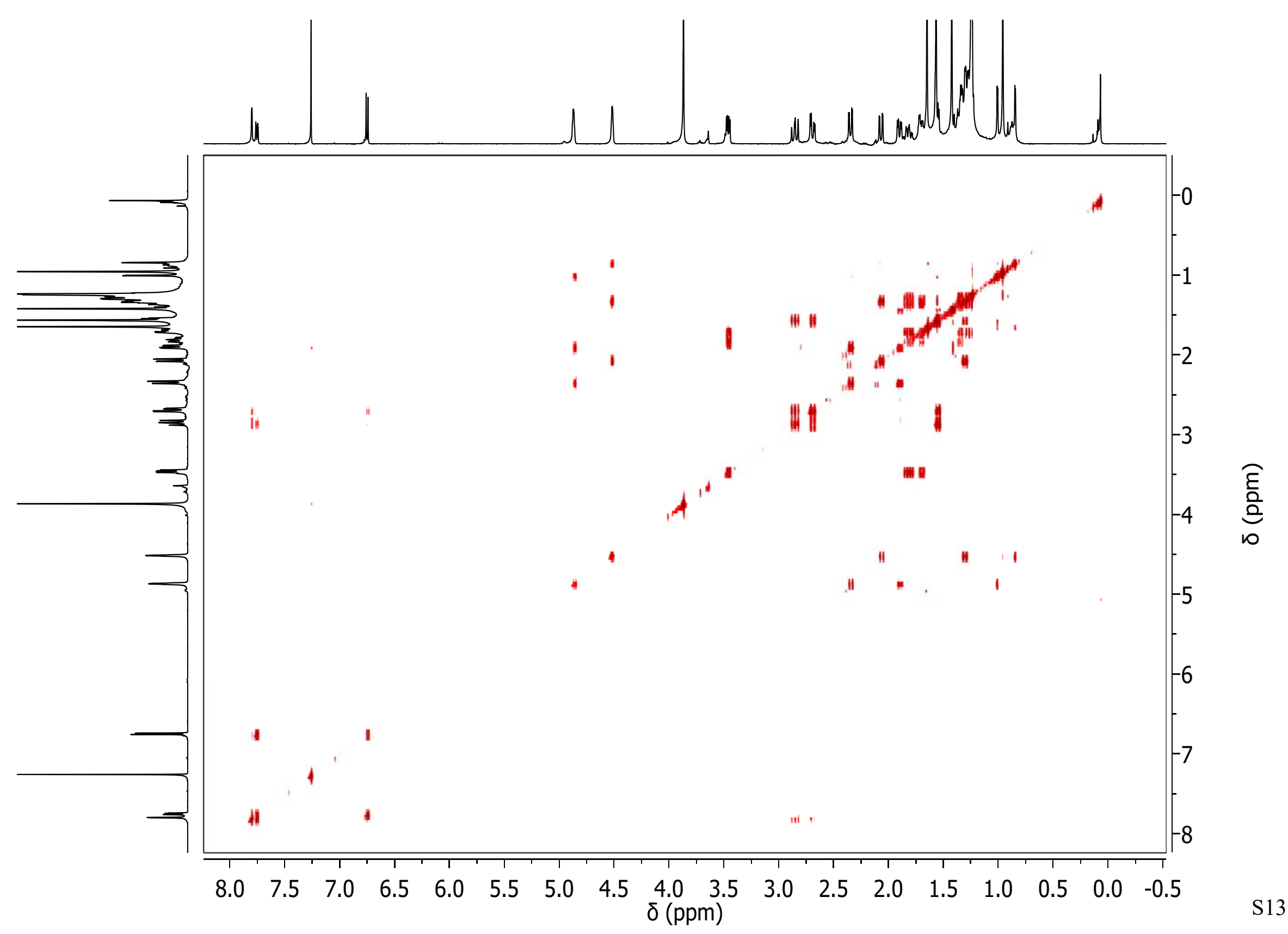


Figure S11. gHMBCAD Spectrum of $2\left(\mathrm{CDCl}_{3}, 500 \mathrm{MHz}\right)$

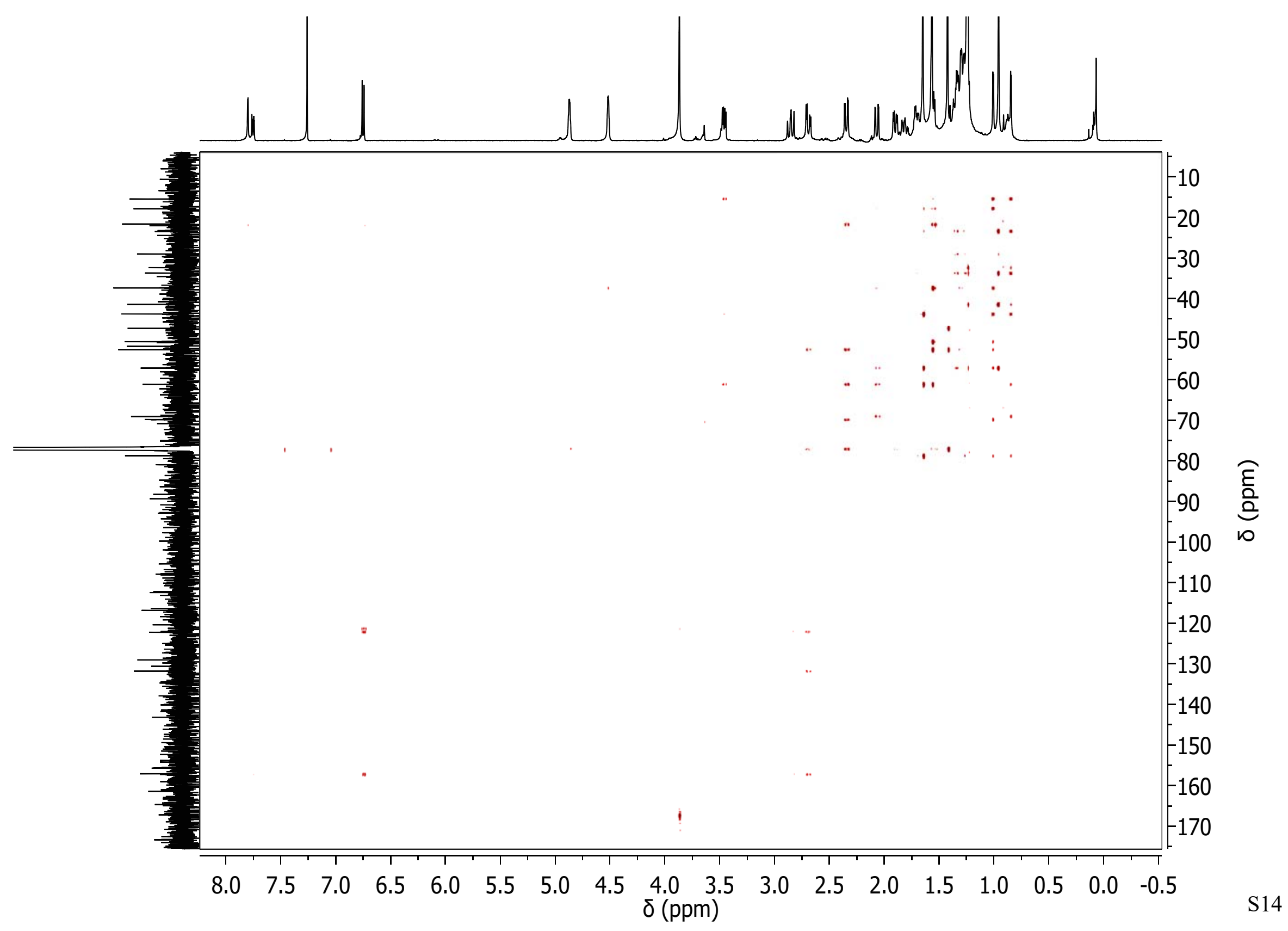


Figure S12. 1D-TOCSY Spectrum of $2\left(\mathrm{CDCl}_{3}, 500 \mathrm{MHz}\right)$ irradiating resonance at $2.85 \mathrm{ppm}$

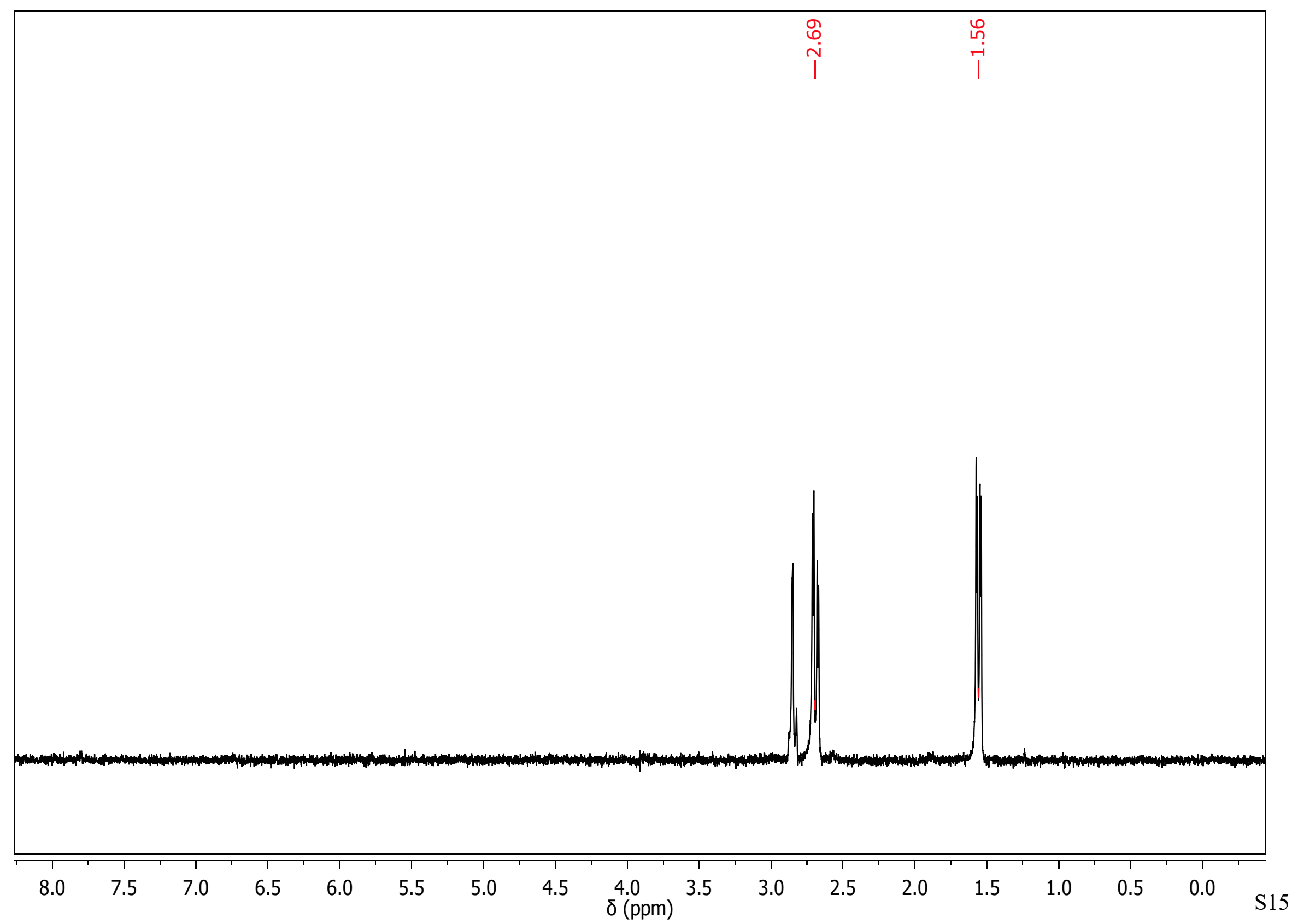


Figure S13. 1D-TOCSY Spectrum of $2\left(\mathrm{CDCl}_{3}, 500 \mathrm{MHz}\right)$ irradiating resonance at $2.69 \mathrm{ppm}$

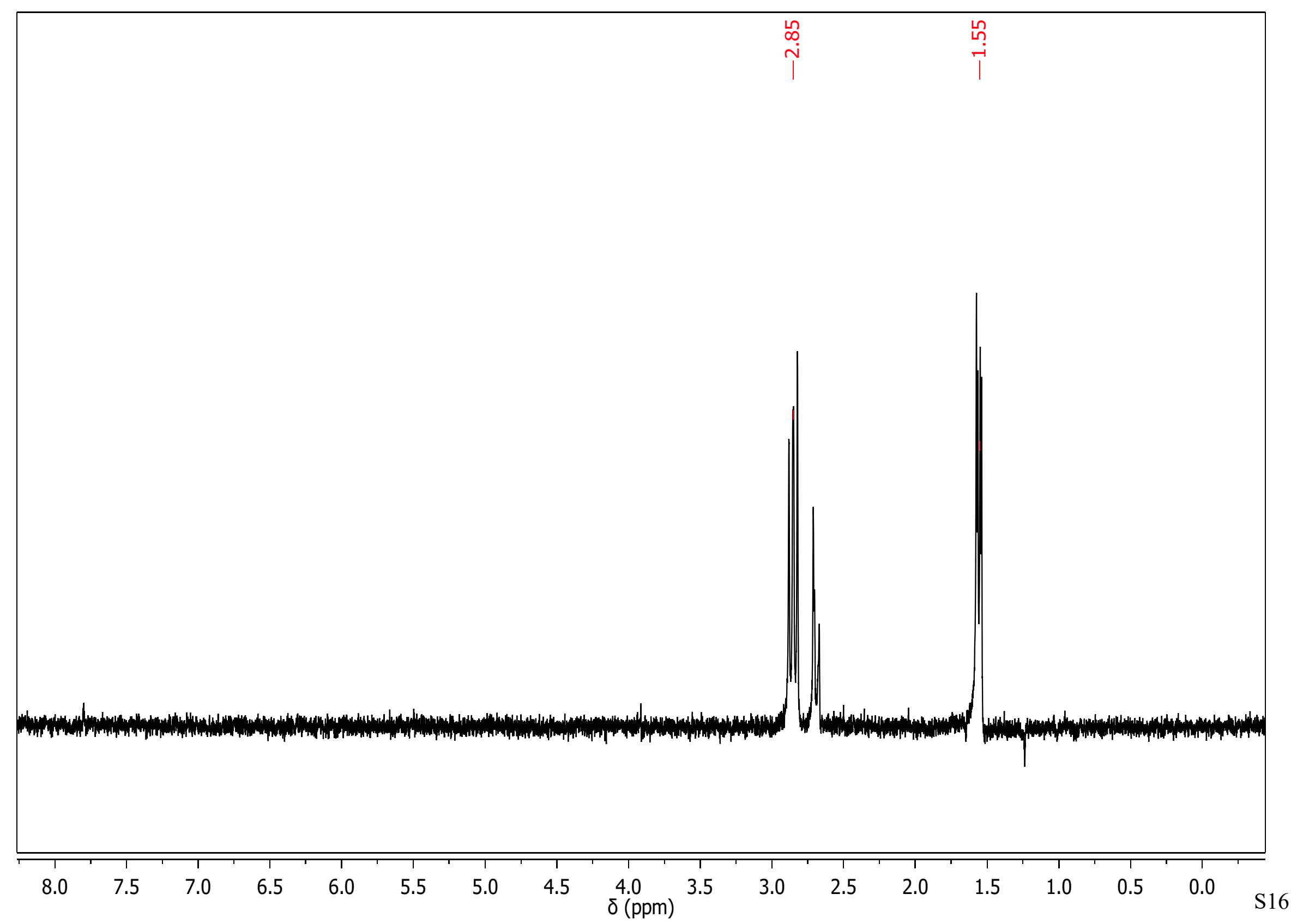


Figure S14. 1D-TOCSY Spectrum of $2\left(\mathrm{CDCl}_{3}, 500 \mathrm{MHz}\right)$ irradiating resonance at $2.06 \mathrm{ppm}$

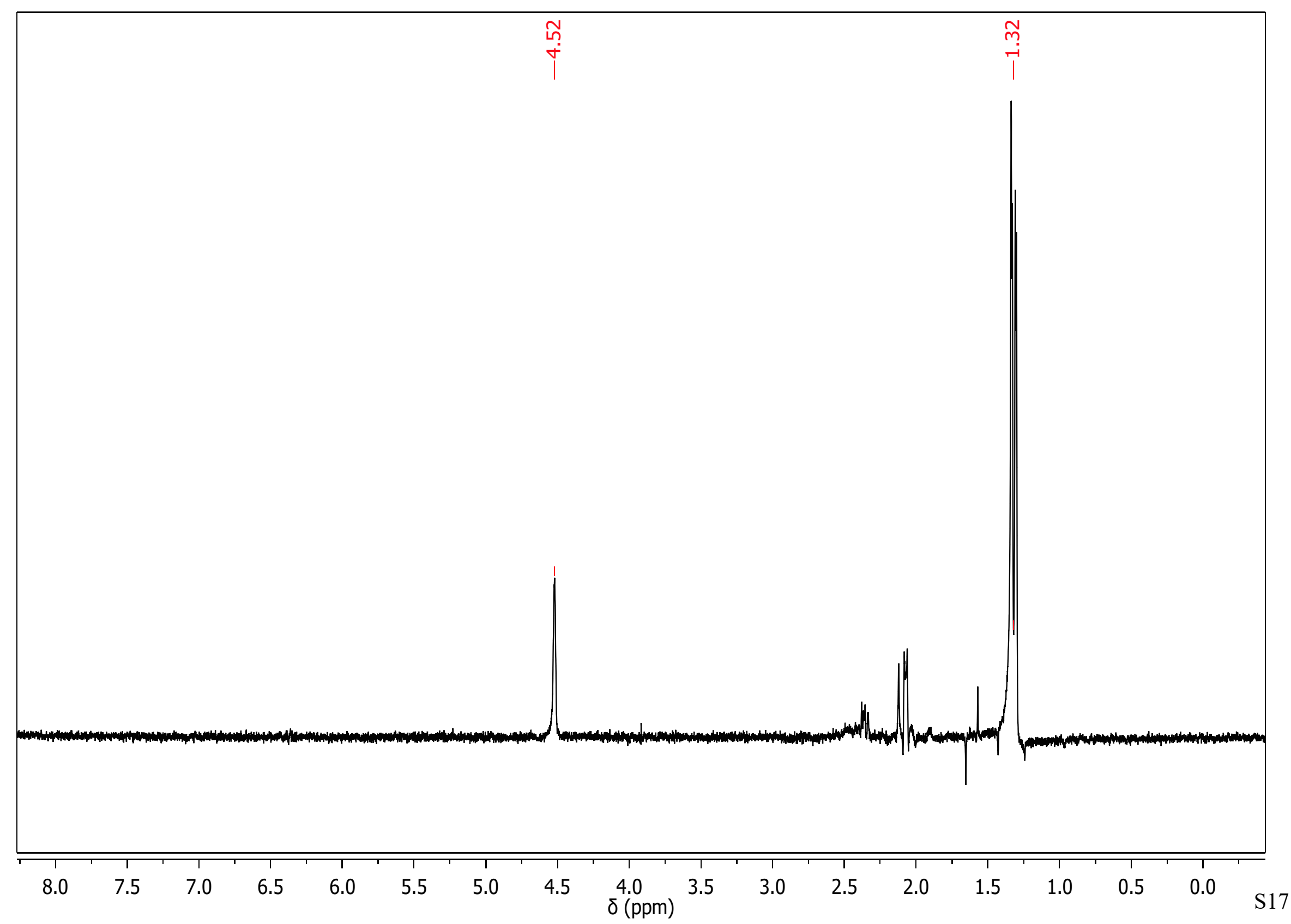


Table S1. Optimized Conformers of 1 - Energy and Abundance.

\begin{tabular}{|c|c|c|c|c|c|c|}
\hline Conf. & Filename & $\begin{array}{l}\text { Free Energy } \\
(\mathrm{kcal} / \mathrm{mol})\end{array}$ & $\begin{array}{l}\text { Relative Energy } \\
\qquad(\mathrm{kcal} / \mathrm{mol})\end{array}$ & Boltzmann Factor & $\begin{array}{c}\text { Equilibrium Mole } \\
\text { Fraction }\end{array}$ & $\begin{array}{l}\text { Number of } \\
\text { Imaginary } \\
\text { Frequencies }\end{array}$ \\
\hline 6 & 1a-opt_freq-conf-6.log & $\begin{array}{c}- \\
872500.7898\end{array}$ & 0 & 0 & 0.331677423 & 0 \\
\hline 5 & 1a-opt_freq-conf-5.log & $\begin{array}{c}- \\
872500.6668\end{array}$ & 0.122991841 & 0.122991841 & 0.269440076 & 0 \\
\hline 1 & 1a-opt_freq-conf-1.log & $\begin{array}{c}- \\
872500.4823\end{array}$ & 0.307479602 & 0.307479602 & 0.197279344 & 0 \\
\hline 2 & 1a-opt_freq-conf-2.log & $\begin{array}{c}- \\
872500.4616\end{array}$ & 0.328187412 & 0.328187412 & 0.190495989 & 0 \\
\hline 3 & 1a-opt_freq-conf-3.log & $\begin{array}{c}- \\
872498.2503\end{array}$ & 2.539530505 & 2.539530505 & 0.004541097 & 0 \\
\hline 4 & 1a-opt_freq-conf-4.log & $\begin{array}{c}- \\
872498.2045\end{array}$ & 2.585338691 & 2.585338691 & 0.00420287 & 0 \\
\hline 7 & 1a-opt_freq-conf-7.log & $\begin{array}{c}- \\
872497.4445\end{array}$ & 3.345252563 & 3.345252563 & 0.001163876 & 0 \\
\hline 8 & 1a-opt_freq-conf-8.log & -872497.068 & 3.721758198 & 3.721758198 & 0.000616056 & 0 \\
\hline 10 & 1a-opt_freq-conf-10.log & $\begin{array}{c}- \\
872496.6476\end{array}$ & 4.14218949 & 4.14218949 & 0.000302761 & 0 \\
\hline 9 & 1a-opt_freq-conf-9.log & $\begin{array}{c}- \\
872496.6024\end{array}$ & 4.187370166 & 4.187370166 & 0.000280508 & 0 \\
\hline
\end{tabular}



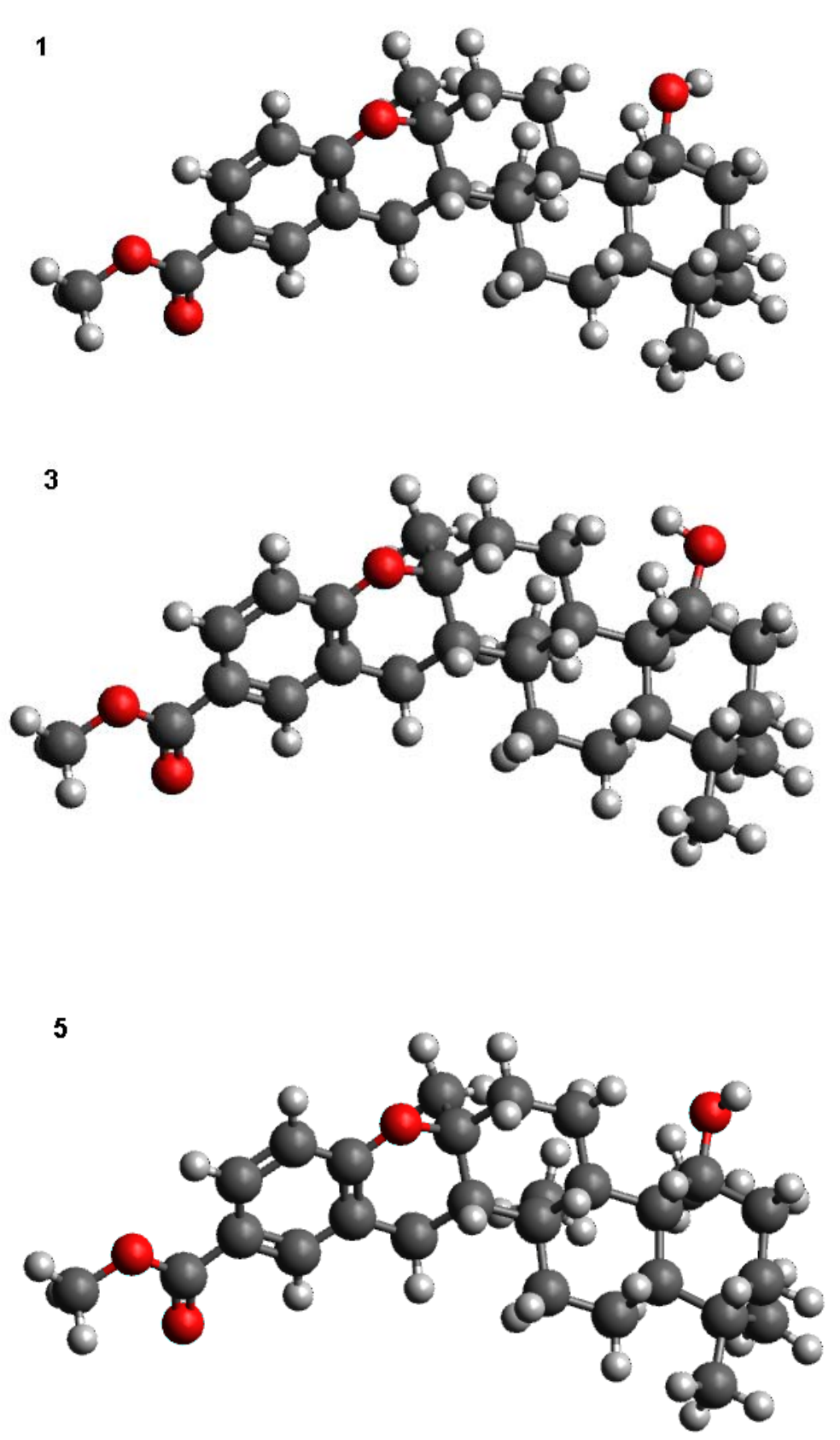
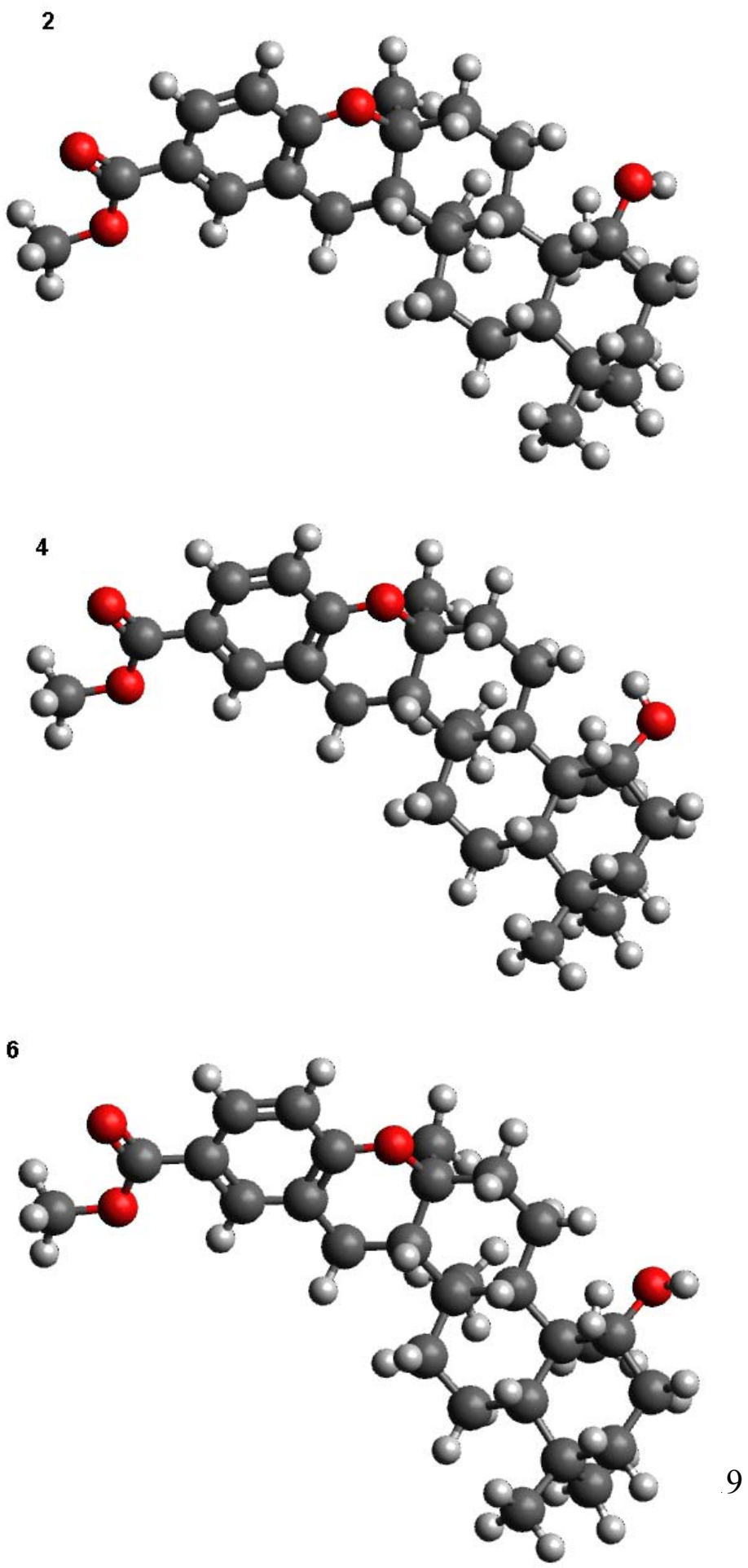

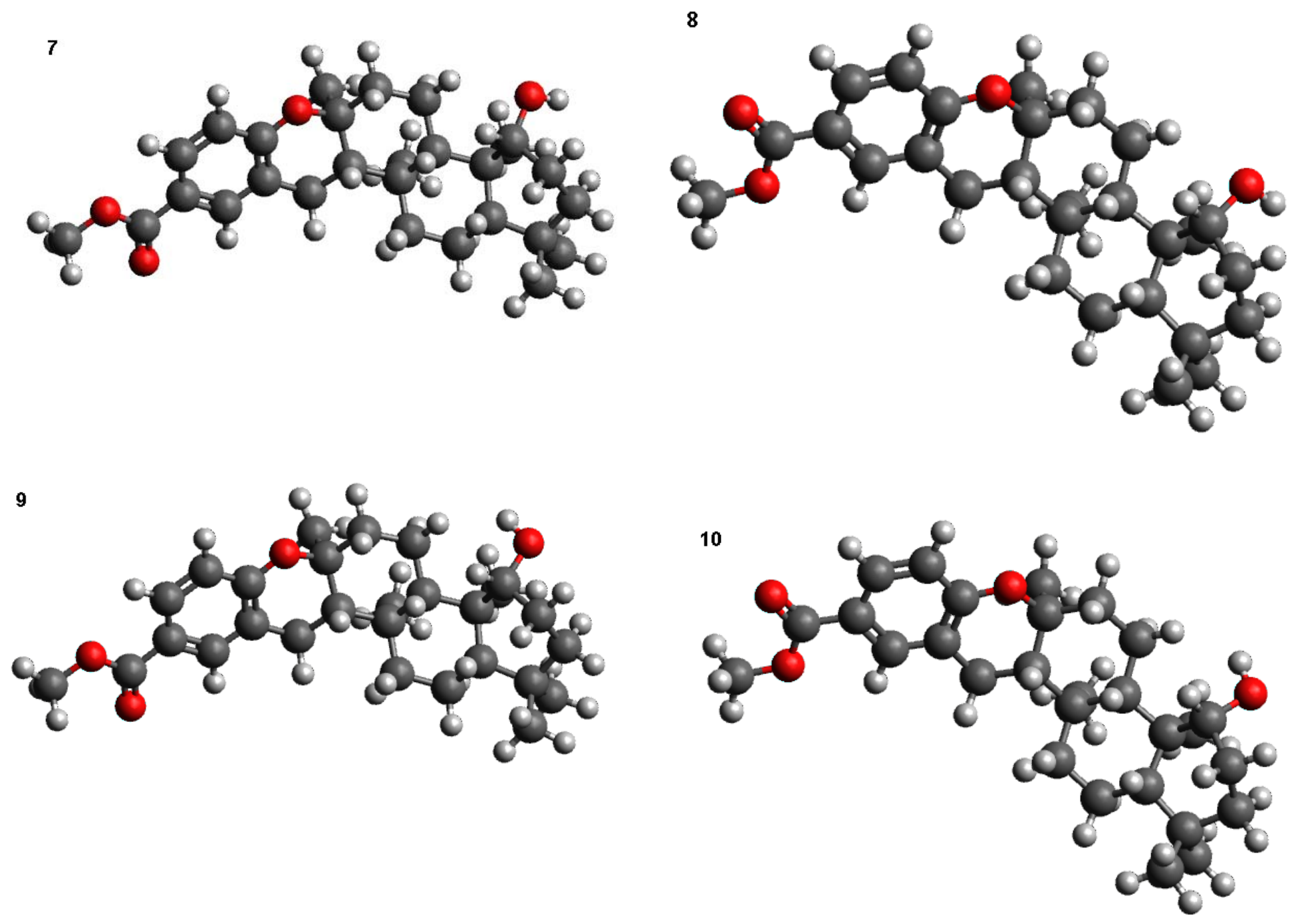
Table S2. Cartesian Coordinates For The Re-Optimized Conformer 1 of Compound 1

1a-opt_freq-conf-1.log

Energy: -872862.6243373 kcal/mol

\begin{tabular}{lccc} 
Atom & $\mathrm{X}(\AA)$ & $\mathrm{Y}$ & $\mathrm{Z}$ \\
$\mathrm{C}$ & -8.80713 & 1.56549 & -0.60377 \\
$\mathrm{O}$ & -7.62301 & 0.77420 & -0.69854 \\
$\mathrm{C}$ & -6.52545 & 1.25186 & -0.08045 \\
$\mathrm{O}$ & -6.52795 & 2.30276 & 0.53131 \\
$\mathrm{C}$ & -5.34631 & 0.36344 & -0.23361 \\
$\mathrm{C}$ & -5.39370 & -0.84718 & -0.95196 \\
$\mathrm{C}$ & -4.25745 & -1.63790 & -1.05838 \\
$\mathrm{C}$ & -3.05129 & -1.23847 & -0.45041 \\
$\mathrm{C}$ & -2.97790 & -0.02134 & 0.26210 \\
$\mathrm{C}$ & -4.13445 & 0.75525 & 0.35667 \\
$\mathrm{O}$ & -1.98791 & -2.05451 & -0.60833 \\
$\mathrm{C}$ & -0.80898 & -1.86735 & 0.23800 \\
$\mathrm{C}$ & -0.48456 & -0.35054 & 0.26625 \\
$\mathrm{C}$ & -1.66604 & 0.42229 & 0.87202 \\
$\mathrm{C}$ & 0.30927 & -2.61949 & -0.48669 \\
$\mathrm{C}$ & 1.70609 & -2.27624 & 0.03891 \\
$\mathrm{C}$ & 1.98556 & -0.76219 & -0.05602 \\
$\mathrm{C}$ & 0.93947 & 0.03393 & 0.81449 \\
$\mathrm{C}$ & 3.50153 & -0.32085 & 0.07945 \\
$\mathrm{C}$ & 3.52498 & 1.22541 & -0.24735 \\
$\mathrm{C}$ & 2.59788 & 2.00112 & 0.69952 \\
$\mathrm{C}$ & 1.14343 & 1.54819 & 0.56900 \\
$\mathrm{C}$ & 4.35440 & -1.03695 & -1.02982 \\
$\mathrm{C}$ & 5.73053 & -0.39919 & -1.24688 \\
$\mathrm{C}$ & 5.66930 & 1.09182 & -1.55037 \\
$\mathrm{C}$ & 4.91903 & 1.90934 & -0.47535 \\
$\mathrm{C}$ & -1.14476 & -2.51849 & 1.58621 \\
$\mathrm{H}$ & -0.45373 & -0.07300 & -0.80308 \\
$\mathrm{H}$ & 1.81703 & -2.63147 & 1.07718 \\
$\mathrm{C}$ & 1.06208 & -0.22623 & 2.33553 \\
$\mathrm{H}$ & 2.93927 & 1.90633 & 1.74325 \\
$\mathrm{H}$ & 3.04784 & 1.28897 & -1.24471 \\
$\mathrm{C}$ & 4.71234 & 3.32800 & -1.05192 \\
$\mathrm{C}$ & 5.77643 & 2.05211 & 0.79923 \\
& 4.09028 & -0.68992 & 1.45983 \\
$\mathrm{H}$ & 4.52935 & -2.43705 & -0.82834 \\
\hline & -9.58257 & 1.02437 & -1.15984
\end{tabular}

$\begin{array}{rrrr} & & & \\ \mathrm{H} & -9.11195 & 1.69189 & 0.44637 \\ \mathrm{H} & -8.64987 & 2.56185 & -1.04442 \\ \mathrm{H} & -6.32650 & -1.16385 & -1.42163 \\ \mathrm{H} & -4.27326 & -2.58276 & -1.60735 \\ \mathrm{H} & -4.10197 & 1.70478 & 0.89820 \\ \mathrm{H} & -1.54691 & 1.50276 & 0.70339 \\ \mathrm{H} & -1.70650 & 0.29164 & 1.96739 \\ \mathrm{H} & 0.12261 & -3.70466 & -0.42400 \\ \mathrm{H} & 0.24653 & -2.34913 & -1.55401 \\ \mathrm{H} & 2.45137 & -2.83247 & -0.53856 \\ \mathrm{H} & 2.64164 & 3.07649 & 0.47319 \\ \mathrm{H} & 0.79389 & 1.79474 & -0.44974 \\ \mathrm{H} & 0.51518 & 2.13087 & 1.26290 \\ \mathrm{H} & 3.78662 & -0.95466 & -1.97441 \\ \mathrm{H} & 6.35628 & -0.58158 & -0.35447 \\ \mathrm{H} & 6.22077 & -0.94137 & -2.07218 \\ \mathrm{H} & 6.68849 & 1.49512 & -1.68086 \\ \mathrm{H} & 5.15544 & 1.23648 & -2.51797 \\ \mathrm{H} & -0.26197 & -2.60695 & 2.23070 \\ \mathrm{H} & -1.91498 & -1.96261 & 2.14009 \\ \mathrm{H} & -1.53018 & -3.53197 & 1.39789 \\ \mathrm{H} & 1.85537 & 0.38451 & 2.78416 \\ \mathrm{H} & 0.13185 & 0.04359 & 2.85693 \\ \mathrm{H} & 1.28744 & -1.27055 & 2.58486 \\ \mathrm{H} & 5.67333 & 3.73046 & -1.41218 \\ \mathrm{H} & 4.01392 & 3.31987 & -1.90510 \\ \mathrm{H} & 4.32646 & 4.03669 & -0.30367 \\ \mathrm{H} & 6.67312 & 2.65531 & 0.58015 \\ \mathrm{H} & 5.22480 & 2.56806 & 1.60072 \\ \mathrm{H} & 6.12292 & 1.09149 & 1.20143 \\ \mathrm{H} & 5.18808 & -0.74670 & 1.43783 \\ \mathrm{H} & 3.83316 & 0.03263 & 2.24306 \\ \mathrm{H} & 3.73020 & -1.67403 & 1.79158 \\ \mathrm{H} & 5.21095 & -2.57500 & -0.14984 \\ & & & \\ & & & \end{array}$

Table S3. Cartesian Coordinates For The Re-Optimized Conformer 2 of Compound 1

1a-opt_freq-conf-2.log

Energy: $-872862.5981200 \mathrm{kcal} / \mathrm{mol}$

$\begin{array}{lccc}\text { Atom } & \mathrm{X}(\AA) & \mathrm{Y} & \mathrm{Z} \\ \mathrm{C} & -7.68096 & 2.92821 & 0.29836 \\ \mathrm{O} & -6.51920 & 2.10120 & 0.24007\end{array}$




\begin{tabular}{|c|c|c|c|c|c|c|c|}
\hline $\mathrm{C}$ & -6.64295 & 0.92913 & -0.41316 & $\mathrm{H}$ & 2.49619 & 3.09972 & 0.35627 \\
\hline $\mathrm{O}$ & -7.68623 & 0.57886 & -0.93012 & $\mathrm{H}$ & 0.70379 & 1.72639 & -0.54632 \\
\hline $\mathrm{C}$ & -5.38638 & 0.13932 & -0.42569 & $\mathrm{H}$ & 0.38095 & 2.12893 & 1.14417 \\
\hline $\mathrm{C}$ & -5.38171 & -1.10685 & -1.08108 & $\mathrm{H}$ & 3.79139 & -0.98412 & -1.90389 \\
\hline $\mathrm{C}$ & -4.22736 & -1.87513 & -1.11664 & $\mathrm{H}$ & 6.32330 & -0.48604 & -0.25776 \\
\hline $\mathrm{C}$ & -3.04962 & -1.41473 & -0.49543 & $\mathrm{H}$ & 6.22710 & -0.91581 & -1.96200 \\
\hline $\mathrm{C}$ & -3.02732 & -0.16312 & 0.15771 & $\mathrm{H}$ & 6.62439 & 1.54644 & -1.65833 \\
\hline $\mathrm{C}$ & -4.20327 & 0.59188 & 0.18049 & $\mathrm{H}$ & 5.11550 & 1.21258 & -2.51247 \\
\hline $\mathrm{O}$ & -1.96276 & -2.20961 & -0.58447 & $\mathrm{H}$ & -0.28819 & -2.57851 & 2.31406 \\
\hline $\mathrm{C}$ & -0.80920 & -1.94860 & 0.27679 & $\mathrm{H}$ & -1.95568 & -1.98629 & 2.15664 \\
\hline $\mathrm{C}$ & -0.52782 & -0.42328 & 0.23977 & $\mathrm{H}$ & -1.51090 & -3.57701 & 1.49829 \\
\hline $\mathrm{C}$ & -1.74444 & 0.34222 & 0.78195 & $\mathrm{H}$ & 1.74045 & 0.49172 & 2.76561 \\
\hline $\mathrm{C}$ & 0.34516 & -2.70185 & -0.38763 & $\mathrm{H}$ & 0.02535 & 0.10569 & 2.82254 \\
\hline $\mathrm{C}$ & 1.71992 & -2.29218 & 0.14756 & $\mathrm{H}$ & 1.22202 & -1.18592 & 2.63142 \\
\hline $\mathrm{C}$ & 1.95756 & -0.77664 & -0.01628 & $\mathrm{H}$ & 5.53988 & 3.76093 & -1.50509 \\
\hline $\mathrm{C}$ & 0.87291 & 0.02763 & 0.79778 & $\mathrm{H}$ & 3.90171 & 3.28175 & -2.00688 \\
\hline $\mathrm{C}$ & 3.45795 & -0.28564 & 0.12108 & $\mathrm{H}$ & 4.16570 & 4.07466 & -0.43279 \\
\hline $\mathrm{C}$ & 3.44330 & 1.24592 & -0.26975 & $\mathrm{H}$ & 6.53469 & 2.79918 & 0.54865 \\
\hline $\mathrm{C}$ & 2.47764 & 2.03392 & 0.62696 & $\mathrm{H}$ & 5.07080 & 2.71483 & 1.54711 \\
\hline $\mathrm{C}$ & 1.03914 & 1.53470 & 0.48888 & $\mathrm{H}$ & 6.01569 & 1.24825 & 1.22694 \\
\hline $\mathrm{C}$ & 4.34564 & -1.01915 & -0.94842 & $\mathrm{H}$ & 5.13696 & -0.60485 & 1.51874 \\
\hline $\mathrm{C}$ & 5.70876 & -0.35507 & -1.16682 & $\mathrm{H}$ & 3.74448 & 0.15839 & 2.27422 \\
\hline $\mathrm{C}$ & 5.61402 & 1.12074 & -1.53048 & $\mathrm{H}$ & 3.70402 & -1.56610 & 1.88536 \\
\hline $\mathrm{C}$ & 4.82121 & 1.95988 & -0.50358 & $\mathrm{H}$ & 5.22170 & -2.50429 & 0.00089 \\
\hline $\mathrm{C}$ & -1.15781 & -2.54532 & 1.64681 & & & & \\
\hline $\mathrm{H}$ & -0.48088 & -0.19544 & -0.84066 & & & & \\
\hline $\mathrm{H}$ & 1.81859 & -2.59284 & 1.20409 & \multirow{3}{*}{\multicolumn{4}{|c|}{$\begin{array}{l}\text { Table S4. Cartesian Coordinates Fo } \\
\text { The Re-Optimized Conformer } 3 \text { of }\end{array}$}} \\
\hline $\mathrm{C}$ & 0.97278 & -0.16066 & 2.33098 & & & & \\
\hline $\mathrm{H}$ & 2.80151 & 1.99243 & 1.67977 & & & & \\
\hline $\mathrm{H}$ & 2.98232 & 1.25343 & -1.27670 & \multirow{2}{*}{\multicolumn{4}{|c|}{ Compound 1}} \\
\hline $\mathrm{C}$ & 4.58455 & 3.34637 & -1.14354 & & & & \\
\hline $\mathrm{C}$ & 5.65091 & 2.18096 & 0.77809 & \\
\hline $\mathrm{C}$ & 4.03774 & -0.58368 & 1.52241 & \multirow{2}{*}{\multicolumn{4}{|c|}{$\begin{array}{l}\text { 1a-opt_freq-conf-3.log } \\
\text { Energy: }-872860.1213025 \mathrm{kcal} / \mathrm{mol}\end{array}$}} \\
\hline $\mathrm{O}$ & 4.55103 & -2.40609 & -0.69534 & & & & \\
\hline $\mathrm{H}$ & 1.71699 & -0.55662 & -1.07371 & & & & 7 \\
\hline $\mathrm{H}$ & -8.50659 & 2.41010 & 0.80976 & Atom & $\begin{array}{c}X(\mathrm{~A}) \\
882414\end{array}$ & Y & $\begin{array}{c}Z \\
05452\end{array}$ \\
\hline $\mathrm{H}$ & -7.39119 & 3.82441 & 0.86068 & C & -8.82414 & 1.55842 & -0.54525 \\
\hline $\mathrm{H}$ & -8.01589 & 3.20667 & -0.71256 & $\mathrm{O}$ & -7.63960 & 0.76968 & -0.65568 \\
\hline $\mathrm{H}$ & -6.29917 & -1.45921 & -1.55772 & $\mathrm{C}$ & -6.53599 & 1.24758 & -0.04909 \\
\hline $\mathrm{H}$ & -4.20448 & -2.84598 & -1.61789 & $\mathrm{O}$ & -6.53282 & 2.29655 & 0.56578 \\
\hline $\mathrm{H}$ & -4.19657 & 1.56563 & 0.67589 & $\mathrm{C}$ & -5.35706 & 0.36141 & -0.21865 \\
\hline $\mathrm{H}$ & -1.64923 & 1.41733 & 0.56963 & $\mathrm{C}$ & -5.41098 & -0.84584 & -0.94208 \\
\hline $\mathrm{H}$ & -1.80990 & 0.25721 & 1.88070 & $\mathrm{C}$ & -4.27489 & -1.63441 & -1.06500 \\
\hline $\mathrm{H}$ & 0.18823 & -3.78770 & -0.27510 & $\mathrm{C}$ & -3.06334 & -1.23538 & -0.46837 \\
\hline $\mathrm{H}$ & 0.29719 & -2.48587 & -1.46798 & $\mathrm{C}$ & -2.98340 & -0.02285 & 0.24988 \\
\hline $\mathrm{H}$ & 2.49292 & -2.85291 & -0.38739 & $\mathrm{C}$ & -4.13973 & 0.75214 & 0.36073 \\
\hline & & & & $\mathrm{O}$ & -1.99954 & -2.04840 & -0.64358 \\
\hline
\end{tabular}




\begin{tabular}{|c|c|c|c|c|c|c|c|}
\hline $\mathrm{C}$ & -0.81122 & -1.86509 & 0.18732 & $\mathrm{H}$ & -1.87311 & -1.99401 & 2.11095 \\
\hline $\mathrm{C}$ & -0.48810 & -0.35031 & 0.23146 & $\mathrm{H}$ & -1.51195 & -3.54998 & 1.32898 \\
\hline $\mathrm{C}$ & -1.66648 & 0.41690 & 0.85124 & $\mathrm{H}$ & 1.82365 & 0.36860 & 2.76851 \\
\hline $\mathrm{C}$ & 0.29488 & -2.59322 & -0.57592 & $\mathrm{H}$ & 0.11173 & -0.02699 & 2.81956 \\
\hline $\mathrm{C}$ & 1.70805 & -2.26419 & -0.07960 & $\mathrm{H}$ & 1.30968 & -1.29937 & 2.53516 \\
\hline $\mathrm{C}$ & 1.99563 & -0.74922 & -0.09191 & $\mathrm{H}$ & 5.66014 & 3.72028 & -1.47016 \\
\hline $\mathrm{C}$ & 0.93683 & 0.02398 & 0.78428 & $\mathrm{H}$ & 4.01285 & 3.27786 & -1.97543 \\
\hline $\mathrm{C}$ & 3.51099 & -0.31284 & 0.10304 & $\mathrm{H}$ & 4.29402 & 4.03874 & -0.38862 \\
\hline $\mathrm{C}$ & 3.52598 & 1.22584 & -0.26092 & $\mathrm{H}$ & 6.67079 & 2.67365 & 0.54378 \\
\hline $\mathrm{C}$ & 2.58805 & 2.00904 & 0.67028 & $\mathrm{H}$ & 5.21080 & 2.65188 & 1.55120 \\
\hline $\mathrm{C}$ & 1.13671 & 1.54206 & 0.54992 & $\mathrm{H}$ & 6.08528 & 1.14444 & 1.22084 \\
\hline $\mathrm{C}$ & 4.41948 & -1.07631 & -0.92927 & $\mathrm{H}$ & 5.14907 & -0.72947 & 1.50603 \\
\hline $\mathrm{C}$ & 5.77032 & -0.40050 & -1.17470 & $\mathrm{H}$ & 3.80190 & 0.13125 & 2.25739 \\
\hline $\mathrm{C}$ & 5.66918 & 1.07035 & -1.54721 & $\mathrm{H}$ & 3.67030 & -1.59343 & 1.88468 \\
\hline $\mathrm{C}$ & 4.91332 & 1.91498 & -0.49813 & $\mathrm{H}$ & 3.99050 & -2.93444 & -0.39298 \\
\hline $\mathrm{C}$ & -1.11799 & -2.54215 & 1.52922 & & & & \\
\hline $\mathrm{H}$ & -0.46019 & -0.05997 & -0.83454 & \multirow{3}{*}{\multicolumn{4}{|c|}{$\begin{array}{l}\text { Table S5. Cartesian Coordinates Fo } \\
\text { The Re-Optimized Conformer } 4 \text { of }\end{array}$}} \\
\hline $\mathrm{H}$ & 1.88130 & -2.70347 & 0.91713 & & & & \\
\hline $\mathrm{C}$ & 1.05409 & -0.25798 & 2.30214 & & & & \\
\hline $\mathrm{H}$ & 2.93008 & 1.93883 & 1.71546 & \multirow{2}{*}{\multicolumn{4}{|c|}{ Compound 1}} \\
\hline $\mathrm{H}$ & 3.05395 & 1.26638 & -1.26141 & & & & \\
\hline $\mathrm{C}$ & 4.69885 & 3.31582 & -1.11296 & \multicolumn{4}{|c|}{ 1a-ont frea-conf-4 loo } \\
\hline $\mathrm{C}$ & 5.76177 & 2.09464 & 0.77726 & \multirow{2}{*}{\multicolumn{4}{|c|}{$\begin{array}{l}\text { la-opt_freq-cont-4.log } \\
\text { Energy: }-872860.0770254 \mathrm{kcal} / \mathrm{mol}\end{array}$}} \\
\hline $\mathrm{C}$ & 4.05593 & -0.63332 & 1.51492 & & & & \\
\hline $\mathrm{O}$ & 4.77877 & -2.39379 & -0.52118 & Atom & $X(\AA)$ & $\mathrm{Y}$ & 7 \\
\hline $\mathrm{H}$ & 1.77137 & -0.43214 & -1.12742 & $\begin{array}{l}\text { Atom } \\
C\end{array}$ & $\begin{array}{c}X(A) \\
-769755\end{array}$ & $\begin{array}{c}Y \\
290982\end{array}$ & $\begin{array}{c}L \\
037047\end{array}$ \\
\hline $\mathrm{H}$ & -9.60498 & 1.01679 & -1.09320 & C & $\begin{array}{r}-1.69 / 55 \\
652303\end{array}$ & 2.90982 & 0.37047 \\
\hline $\mathrm{H}$ & -9.11666 & 1.68202 & 0.50868 & $\mathrm{O}$ & -6.53293 & 2.08891 & 0.28648 \\
\hline $\mathrm{H}$ & -8.67404 & 2.55590 & -0.98582 & $\mathrm{C}$ & -6.66233 & 0.92063 & -0.37198 \\
\hline $\mathrm{H}$ & -6.34847 & -1.16151 & -1.40294 & $\mathrm{O}$ & -7.71220 & 0.56848 & -0.87388 \\
\hline $\mathrm{H}$ & -4.29516 & -2.57648 & -1.61855 & $\mathrm{C}$ & -5.40248 & 0.13628 & -0.40952 \\
\hline $\mathrm{H}$ & -4.10277 & 1.69884 & 0.90687 & $\mathrm{C}$ & -5.40384 & -1.10735 & -1.06964 \\
\hline $\mathrm{H}$ & -1.54935 & 1.49856 & 0.68987 & $\mathrm{C}$ & -4.24762 & -1.87170 & -1.12580 \\
\hline $\mathrm{H}$ & -1.69905 & 0.27816 & 1.94578 & $\mathrm{C}$ & -3.06278 & -1.40891 & -0.52080 \\
\hline $\mathrm{H}$ & 0.11655 & -3.68061 & -0.54082 & $\mathrm{C}$ & -3.03391 & -0.15990 & 0.13537 \\
\hline $\mathrm{H}$ & 0.20986 & -2.29195 & -1.63277 & $\mathrm{C}$ & -4.21181 & 0.59128 & 0.17941 \\
\hline $\mathrm{H}$ & 2.37920 & -2.78675 & -0.77730 & $\mathrm{O}$ & -1.97390 & -2.20018 & -0.62780 \\
\hline $\mathrm{H}$ & 2.61980 & 3.08038 & 0.42318 & $\mathrm{C}$ & -0.81204 & -1.94175 & 0.22032 \\
\hline $\mathrm{H}$ & 0.77692 & 1.79235 & -0.46418 & $\mathrm{C}$ & -0.53090 & -0.41818 & 0.19307 \\
\hline $\mathrm{H}$ & 0.50996 & 2.11578 & 1.25236 & $\mathrm{C}$ & -1.74453 & 0.34576 & 0.74513 \\
\hline $\mathrm{H}$ & 3.87045 & -1.10904 & -1.89392 & $\mathrm{C}$ & 0.33149 & -2.67739 & -0.47748 \\
\hline $\mathrm{H}$ & 6.38711 & -0.52933 & -0.27012 & $\mathrm{C}$ & 1.72215 & -2.28322 & 0.03414 \\
\hline $\mathrm{H}$ & 6.28414 & -0.96849 & -1.96762 & $\mathrm{C}$ & 1.96934 & -0.76365 & -0.05286 \\
\hline $\mathrm{H}$ & 6.67839 & 1.48885 & -1.70476 & $\mathrm{C}$ & 0.86980 & 0.02460 & 0.75728 \\
\hline $\mathrm{H}$ & 5.14286 & 1.16579 & -2.51450 & $\mathrm{C}$ & 3.46765 & -0.27590 & 0.15158 \\
\hline $\mathrm{H}$ & -0.22168 & -2.64749 & 2.15223 & $\mathrm{C}$ & 3.44954 & 1.24144 & -0.29340 \\
\hline & & & & $\mathrm{C}$ & 2.47014 & 2.04567 & 0.57507 \\
\hline
\end{tabular}




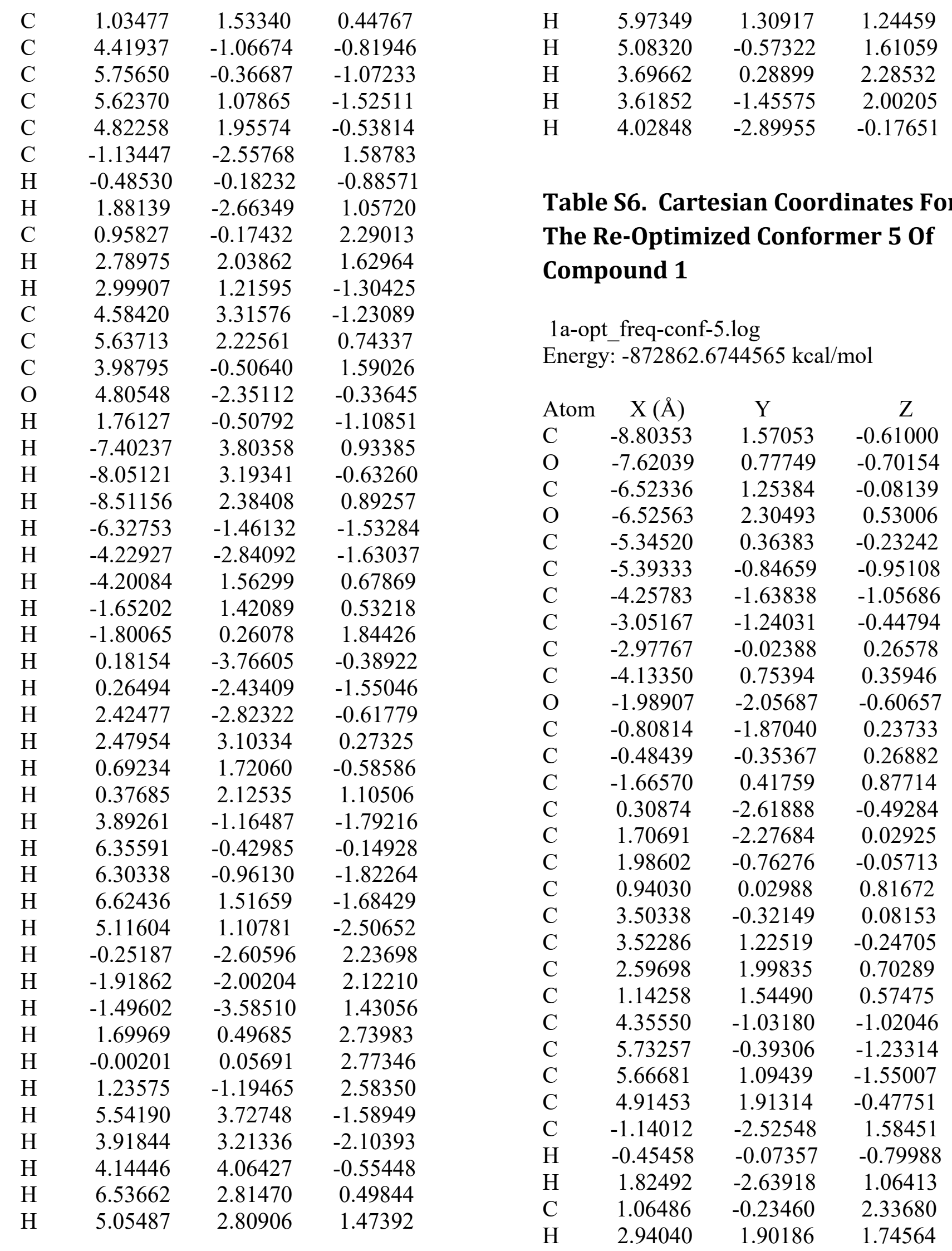




\begin{tabular}{|c|c|c|c|}
\hline $\mathrm{H}$ & 3.04327 & 1.28854 & -1.24310 \\
\hline $\mathrm{C}$ & 4.70348 & 3.32949 & -1.05796 \\
\hline $\mathrm{C}$ & 5.77121 & 2.06045 & 0.79683 \\
\hline $\mathrm{C}$ & 4.09378 & -0.68825 & 1.46283 \\
\hline $\mathrm{O}$ & 4.54146 & -2.40490 & -0.68564 \\
\hline $\mathrm{H}$ & 1.73558 & -0.48030 & -1.09723 \\
\hline $\mathrm{H}$ & -9.57872 & 1.02973 & -1.16676 \\
\hline $\mathrm{H}$ & -9.11012 & 1.69887 & 0.43938 \\
\hline $\mathrm{H}$ & -8.64409 & 2.56604 & -1.05181 \\
\hline $\mathrm{H}$ & -6.32602 & -1.16205 & -1.42173 \\
\hline $\mathrm{H}$ & -4.27406 & -2.58294 & -1.60632 \\
\hline $\mathrm{H}$ & -4.10053 & 1.70325 & 0.90136 \\
\hline $\mathrm{H}$ & -1.54639 & 1.49854 & 0.71189 \\
\hline $\mathrm{H}$ & -1.70601 & 0.28349 & 1.97210 \\
\hline $\mathrm{H}$ & 0.12304 & -3.70448 & -0.43371 \\
\hline $\mathrm{H}$ & 0.24218 & -2.34460 & -1.55896 \\
\hline $\mathrm{H}$ & 2.45072 & -2.82683 & -0.55616 \\
\hline $\mathrm{H}$ & 2.63970 & 3.07406 & 0.47802 \\
\hline $\mathrm{H}$ & 0.79038 & 1.79378 & -0.44254 \\
\hline $\mathrm{H}$ & 0.51558 & 2.12540 & 1.27166 \\
\hline $\mathrm{H}$ & 3.78873 & -0.94753 & -1.97187 \\
\hline $\mathrm{H}$ & 6.34832 & -0.57712 & -0.33759 \\
\hline $\mathrm{H}$ & 6.23298 & -0.93137 & -2.05833 \\
\hline $\mathrm{H}$ & 6.68422 & 1.50107 & -1.68387 \\
\hline $\mathrm{H}$ & 5.15113 & 1.23289 & -2.51776 \\
\hline $\mathrm{H}$ & -0.25549 & -2.61569 & 2.22615 \\
\hline $\mathrm{H}$ & -1.90880 & -1.97107 & 2.14207 \\
\hline $\mathrm{H}$ & -1.52614 & -3.53839 & 1.39436 \\
\hline $\mathrm{H}$ & 1.85822 & 0.37563 & 2.78573 \\
\hline $\mathrm{H}$ & 0.13475 & 0.03203 & 2.86014 \\
\hline $\mathrm{H}$ & 1.29286 & -1.27925 & 2.58207 \\
\hline $\mathrm{H}$ & 5.66369 & 3.73492 & -1.41704 \\
\hline $\mathrm{H}$ & 4.00721 & 3.31633 & -1.91290 \\
\hline $\mathrm{H}$ & 4.31285 & 4.03869 & -0.31265 \\
\hline $\mathrm{H}$ & 6.67196 & 2.65652 & 0.57451 \\
\hline $\mathrm{H}$ & 5.22138 & 2.58609 & 1.59329 \\
\hline $\mathrm{H}$ & 6.10909 & 1.10003 & 1.20623 \\
\hline $\mathrm{H}$ & 5.18839 & -0.76141 & 1.42915 \\
\hline $\mathrm{H}$ & 3.84167 & 0.04192 & 2.24091 \\
\hline $\mathrm{H}$ & 3.73164 & -1.67051 & 1.79400 \\
\hline $\mathrm{H}$ & 5.07880 & -2.81317 & -1.38585 \\
\hline
\end{tabular}

Table S7. Cartesian Coordinates For The Re-Optimized Conformer 6 of Compound 1

\author{
1a-opt_freq-conf-6.log \\ Energy: -872862.6330974 kcal/mol
}

$\begin{array}{lccc}\text { Atom } & \mathrm{X}(\AA) & \mathrm{Y} & \mathrm{Z} \\ \mathrm{C} & -7.68321 & 2.92489 & 0.30738 \\ \mathrm{O} & -6.52099 & 2.09887 & 0.24486 \\ \mathrm{C} & -6.64581 & 0.92689 & -0.40840 \\ \mathrm{O} & -7.69038 & 0.57599 & -0.92233 \\ \mathrm{C} & -5.38864 & 0.13812 & -0.42479 \\ \mathrm{C} & -5.38470 & -1.10823 & -1.07989 \\ \mathrm{C} & -4.22988 & -1.87574 & -1.11814 \\ \mathrm{C} & -3.05085 & -1.41445 & -0.49994 \\ \mathrm{C} & -3.02784 & -0.16249 & 0.15246 \\ \mathrm{C} & -4.20422 & 0.59170 & 0.17805 \\ \mathrm{O} & -1.96372 & -2.20870 & -0.59066 \\ \mathrm{C} & -0.80911 & -1.94742 & 0.26949 \\ \mathrm{C} & -0.52768 & -0.42238 & 0.23218 \\ \mathrm{C} & -1.74412 & 0.34382 & 0.77386 \\ \mathrm{C} & 0.34494 & -2.69937 & -0.39649 \\ \mathrm{C} & 1.72011 & -2.29157 & 0.13892 \\ \mathrm{C} & 1.95939 & -0.77626 & -0.01882 \\ \mathrm{C} & 0.87262 & 0.02872 & 0.79141 \\ \mathrm{C} & 3.46046 & -0.28541 & 0.12886 \\ \mathrm{C} & 3.44454 & 1.24337 & -0.27576 \\ \mathrm{C} & 2.47677 & 2.03565 & 0.61481 \\ \mathrm{C} & 1.03865 & 1.53512 & 0.47865 \\ \mathrm{C} & 4.35562 & -1.02355 & -0.91942 \\ \mathrm{C} & 5.71931 & -0.35743 & -1.13383 \\ \mathrm{C} & 5.62012 & 1.11026 & -1.52534 \\ \mathrm{C} & 4.82126 & 1.95889 & -0.51125 \\ \mathrm{C} & -1.15597 & -2.54441 & 1.63977 \\ \mathrm{H} & -0.48013 & -0.19495 & -0.84831 \\ \mathrm{H} & 1.82259 & -2.59731 & 1.19353 \\ \mathrm{C} & 0.96945 & -0.15721 & 2.32513 \\ \mathrm{H} & 2.79935 & 2.00063 & 1.66807 \\ \mathrm{H} & 2.98557 & 1.24365 & -1.28347 \\ \mathrm{C} & 4.58396 & 3.33792 & -1.16660 \\ \mathrm{C} & 5.64440 & 2.19372 & 0.77201 \\ \mathrm{C} & 4.03043 & -0.56790 & 1.53823 \\ \mathrm{O} & 4.57112 & -2.37337 & -0.51522 \\ \mathrm{H} & 1.72472 & -0.55375 & -1.07699 \\ & -7.39226 & 3.82119 & 0.86894\end{array}$




\begin{tabular}{|c|c|c|c|c|c|c|c|}
\hline $\mathrm{H}$ & -8.02191 & 3.20336 & -0.70228 & $\mathrm{C}$ & -6.53749 & 1.24879 & -0.11864 \\
\hline $\mathrm{H}$ & -8.50664 & 2.40598 & 0.82152 & $\mathrm{O}$ & -6.54166 & 2.31960 & 0.45750 \\
\hline $\mathrm{H}$ & -6.30317 & -1.46147 & -1.55394 & $\mathrm{C}$ & -5.35758 & 0.35618 & -0.23871 \\
\hline $\mathrm{H}$ & -4.20773 & -2.84682 & -1.61899 & $\mathrm{C}$ & -5.40328 & -0.87985 & -0.91245 \\
\hline $\mathrm{H}$ & -4.19693 & 1.56554 & 0.67326 & $\mathrm{C}$ & -4.26584 & -1.67228 & -0.99044 \\
\hline $\mathrm{H}$ & -1.64919 & 1.41858 & 0.55959 & $\mathrm{C}$ & -3.05998 & -1.24895 & -0.39839 \\
\hline $\mathrm{H}$ & -1.80852 & 0.26086 & 1.87279 & $\mathrm{C}$ & -2.98858 & -0.00681 & 0.26976 \\
\hline $\mathrm{H}$ & 0.18773 & -3.78549 & -0.28647 & $\mathrm{C}$ & -4.14634 & 0.77080 & 0.33687 \\
\hline $\mathrm{H}$ & 0.29641 & -2.48092 & -1.47638 & $\mathrm{O}$ & -1.99506 & -2.06856 & -0.52868 \\
\hline $\mathrm{H}$ & 2.49272 & -2.84898 & -0.40030 & $\mathrm{C}$ & -0.81336 & -1.84823 & 0.30479 \\
\hline $\mathrm{H}$ & 2.49501 & 3.09976 & 0.33753 & $\mathrm{C}$ & -0.49585 & -0.32809 & 0.27793 \\
\hline $\mathrm{H}$ & 0.70291 & 1.72362 & -0.55703 & $\mathrm{C}$ & -1.67707 & 0.46040 & 0.86208 \\
\hline $\mathrm{H}$ & 0.38035 & 2.13104 & 1.13234 & $\mathrm{C}$ & 0.30206 & -2.63084 & -0.39720 \\
\hline $\mathrm{H}$ & 3.80810 & -1.00048 & -1.88543 & $\mathrm{C}$ & 1.69918 & -2.26030 & 0.10916 \\
\hline $\mathrm{H}$ & 6.31961 & -0.48019 & -0.21751 & $\mathrm{C}$ & 1.95928 & -0.75001 & -0.04327 \\
\hline $\mathrm{H}$ & 6.25263 & -0.92094 & -1.92070 & $\mathrm{C}$ & 0.92916 & 0.07985 & 0.80359 \\
\hline $\mathrm{H}$ & 6.62874 & 1.53882 & -1.65727 & $\mathrm{C}$ & 3.47559 & -0.33152 & 0.03947 \\
\hline $\mathrm{H}$ & 5.12309 & 1.18634 & -2.50957 & $\mathrm{C}$ & 3.55762 & 1.20330 & -0.27175 \\
\hline $\mathrm{H}$ & -0.28537 & -2.57753 & 2.30568 & $\mathrm{C}$ & 2.61053 & 2.02044 & 0.61937 \\
\hline $\mathrm{H}$ & -1.95315 & -1.98536 & 2.15070 & $\mathrm{C}$ & 1.15149 & 1.57879 & 0.48140 \\
\hline $\mathrm{H}$ & -1.50928 & -3.57609 & 1.49160 & $\mathrm{C}$ & 4.23412 & -1.10105 & -1.12260 \\
\hline $\mathrm{H}$ & 1.73359 & 0.49856 & 2.76044 & $\mathrm{C}$ & 5.29591 & -0.24369 & -1.84100 \\
\hline $\mathrm{H}$ & 0.01977 & 0.10523 & 2.81442 & $\mathrm{C}$ & 5.99269 & 0.74545 & -0.91179 \\
\hline $\mathrm{H}$ & 1.22309 & -1.18110 & 2.62644 & $\mathrm{C}$ & 5.00702 & 1.81791 & -0.37043 \\
\hline $\mathrm{H}$ & 5.54030 & 3.75266 & -1.52537 & $\mathrm{C}$ & -1.14145 & -2.45028 & 1.67772 \\
\hline $\mathrm{H}$ & 3.90780 & 3.26198 & -2.03431 & $\mathrm{H}$ & -0.47239 & -0.08858 & -0.80068 \\
\hline $\mathrm{H}$ & 4.15697 & 4.07174 & -0.46644 & $\mathrm{H}$ & 1.81565 & -2.57245 & 1.16000 \\
\hline $\mathrm{H}$ & 6.53450 & 2.80156 & 0.53895 & $\mathrm{C}$ & 1.05848 & -0.11557 & 2.33356 \\
\hline $\mathrm{H}$ & 5.06311 & 2.74418 & 1.52836 & $\mathrm{H}$ & 2.92530 & 1.96087 & 1.67329 \\
\hline $\mathrm{H}$ & 5.99683 & 1.26451 & 1.23724 & $\mathrm{H}$ & 3.14256 & 1.29030 & -1.29288 \\
\hline $\mathrm{H}$ & 5.12721 & -0.60947 & 1.53148 & $\mathrm{C}$ & 4.96181 & 2.98916 & -1.37708 \\
\hline $\mathrm{H}$ & 3.73963 & 0.19040 & 2.27478 & $\mathrm{C}$ & 5.54586 & 2.36738 & 0.96574 \\
\hline $\mathrm{H}$ & 3.68950 & -1.54381 & 1.90839 & $\mathrm{C}$ & 4.10135 & -0.70450 & 1.40049 \\
\hline \multirow[t]{3}{*}{$\mathrm{H}$} & \multirow[t]{3}{*}{5.13430} & \multirow[t]{3}{*}{-2.79937} & \multirow[t]{3}{*}{-1.18382} & $\mathrm{O}$ & 4.77658 & -2.35117 & -0.70547 \\
\hline & & & & $\mathrm{H}$ & 1.68918 & -0.50867 & -1.08876 \\
\hline & & & & $\mathrm{H}$ & -9.59141 & 0.98531 & -1.19859 \\
\hline \multicolumn{4}{|c|}{ Table S8. Cartesian Coordinates For } & $\mathrm{H}$ & -9.12507 & 1.70699 & 0.38531 \\
\hline \multicolumn{4}{|c|}{ The Re-Optimized Conformer 7 of } & $\mathrm{H}$ & -8.65854 & 2.52562 & -1.13290 \\
\hline \multirow{2}{*}{\multicolumn{4}{|c|}{ Compound 1}} & $\mathrm{H}$ & -6.33565 & -1.21481 & -1.37012 \\
\hline & & & & $\mathrm{H}$ & -4.28049 & -2.63643 & -1.50480 \\
\hline \multirow{2}{*}{\multicolumn{4}{|c|}{ 1a-opt_freq-conf-7.log }} & $\mathrm{H}$ & -4.11503 & 1.73935 & 0.84364 \\
\hline \multirow{2}{*}{\multicolumn{4}{|c|}{ Energy: -872859.5718113 kcal/mol }} & $\mathrm{H}$ & -1.55999 & 1.53518 & 0.65812 \\
\hline & & & & $\mathrm{H}$ & -1.71442 & 0.36528 & 1.96118 \\
\hline \multirow{3}{*}{$\begin{array}{l}\text { Atom } \\
\mathrm{C} \\
\mathrm{O}\end{array}$} & \multirow{2}{*}{$X(\AA)$} & \multirow{2}{*}{$\mathrm{Y}$} & \multirow{2}{*}{$\mathrm{Z}$} & $\mathrm{H}$ & 0.11469 & -3.71207 & -0.28835 \\
\hline & & & & $\mathrm{H}$ & 0.23380 & -2.40384 & -1.47400 \\
\hline & $\begin{array}{l}-8.81738 \\
-7.63317\end{array}$ & $\begin{array}{l}1.54487 \\
0.75060\end{array}$ & $\begin{array}{l}-0.65905 \\
-0.72358\end{array}$ & $\mathrm{H}$ & 2.44976 & -2.83758 & -0.44749 \\
\hline
\end{tabular}




\begin{tabular}{|c|c|c|c|c|c|c|c|}
\hline $\mathrm{H}$ & 2.67865 & 3.08772 & 0.35312 & $\mathrm{C}$ & -0.53960 & -0.39251 & 0.23193 \\
\hline $\mathrm{H}$ & 0.82018 & 1.77312 & -0.55463 & $\mathrm{C}$ & -1.75542 & 0.39357 & 0.74393 \\
\hline $\mathrm{H}$ & 0.51945 & 2.20445 & 1.13321 & $\mathrm{C}$ & 0.33486 & -2.70499 & -0.29836 \\
\hline $\mathrm{H}$ & 3.48136 & -1.38030 & -1.87665 & $\mathrm{C}$ & 1.70943 & -2.26891 & 0.21682 \\
\hline $\mathrm{H}$ & 6.01656 & -0.93355 & -2.30967 & $\mathrm{C}$ & 1.93287 & -0.76203 & -0.00992 \\
\hline $\mathrm{H}$ & 4.82255 & 0.30735 & -2.67033 & $\mathrm{C}$ & 0.86106 & 0.08259 & 0.76769 \\
\hline $\mathrm{H}$ & 6.45764 & 0.19800 & -0.07425 & $\mathrm{C}$ & 3.43552 & -0.29914 & 0.08503 \\
\hline $\mathrm{H}$ & 6.82665 & 1.24442 & -1.43361 & $\mathrm{C}$ & 3.48676 & 1.22014 & -0.30048 \\
\hline $\mathrm{H}$ & -0.25575 & -2.51303 & 2.32141 & $\mathrm{C}$ & 2.49526 & 2.05576 & 0.52249 \\
\hline $\mathrm{H}$ & -1.91051 & -1.87604 & 2.21412 & $\mathrm{C}$ & 1.05227 & 1.56782 & 0.37126 \\
\hline $\mathrm{H}$ & -1.52530 & -3.47083 & 1.52874 & $\mathrm{C}$ & 4.23980 & -1.10230 & -1.02220 \\
\hline $\mathrm{H}$ & 1.84998 & 0.51743 & 2.75431 & $\mathrm{C}$ & 5.30189 & -0.25452 & -1.75161 \\
\hline $\mathrm{H}$ & 0.12913 & 0.16964 & 2.84820 & $\mathrm{C}$ & 5.94901 & 0.79376 & -0.85201 \\
\hline $\mathrm{H}$ & 1.29158 & -1.14786 & 2.62394 & $\mathrm{C}$ & 4.92303 & 1.86651 & -0.39163 \\
\hline $\mathrm{H}$ & 5.93845 & 3.49789 & -1.42697 & $\mathrm{C}$ & -1.15963 & -2.45146 & 1.73293 \\
\hline $\mathrm{H}$ & 4.71328 & 2.64051 & -2.39281 & $\mathrm{H}$ & -0.49572 & -0.21180 & -0.85742 \\
\hline $\mathrm{H}$ & 4.21041 & 3.74291 & -1.09271 & $\mathrm{H}$ & 1.80995 & -2.52206 & 1.28503 \\
\hline $\mathrm{H}$ & 6.58781 & 2.70424 & 0.83421 & $\mathrm{C}$ & 0.95869 & -0.02771 & 2.30850 \\
\hline $\mathrm{H}$ & 4.96803 & 3.23459 & 1.32048 & $\mathrm{H}$ & 2.78331 & 2.05910 & 1.58567 \\
\hline $\mathrm{H}$ & 5.54393 & 1.61107 & 1.76360 & $\mathrm{H}$ & 3.09804 & 1.24523 & -1.33533 \\
\hline $\mathrm{H}$ & 5.19820 & -0.63122 & 1.38596 & $\mathrm{C}$ & 4.87725 & 2.98462 & -1.45699 \\
\hline $\mathrm{H}$ & 3.75423 & -0.05199 & 2.21009 & $\mathrm{C}$ & 5.41131 & 2.49562 & 0.92869 \\
\hline $\mathrm{H}$ & 3.85720 & -1.73855 & 1.67796 & $\mathrm{C}$ & 4.03866 & -0.58925 & 1.47631 \\
\hline $\mathrm{H}$ & 5.58704 & -2.19633 & -0.19369 & $\mathrm{O}$ & 4.79831 & -2.31996 & -0.53707 \\
\hline & & & & $\mathrm{H}$ & 1.68186 & -0.58334 & -1.07255 \\
\hline \multirow{2}{*}{\multicolumn{4}{|c|}{ Table S9. Cartesian Coordinates For }} & $\mathrm{H}$ & -7.41540 & 3.84976 & 0.73032 \\
\hline & & & & $\mathrm{H}$ & -8.05661 & 3.16241 & -0.80698 \\
\hline \multicolumn{4}{|c|}{ The Re-Optimized Conformer 8 of } & $\mathrm{H}$ & -8.52342 & 2.42901 & 0.75418 \\
\hline \multirow{2}{*}{\multicolumn{4}{|c|}{ Compound 1}} & $\mathrm{H}$ & -6.32156 & -1.52724 & -1.47423 \\
\hline & & & & $\mathrm{H}$ & -4.22100 & -2.90615 & -1.49388 \\
\hline \multirow{2}{*}{\multicolumn{4}{|c|}{ 1a-opt_freq-conf-8.log }} & $\mathrm{H}$ & -4.21301 & 1.60111 & 0.60578 \\
\hline \multirow{2}{*}{\multicolumn{4}{|c|}{ Energy: -872859.5913896 kcal/mol }} & $\mathrm{H}$ & -1.66253 & 1.45798 & 0.48139 \\
\hline & & & & $\mathrm{H}$ & -1.81558 & 0.35987 & 1.84566 \\
\hline \multirow{2}{*}{ Atom } & \multirow{2}{*}{$X(\AA)$} & $Y$ & \multirow{2}{*}{ Z } & $\mathrm{H}$ & 0.17413 & -3.78363 & -0.13487 \\
\hline & & Y & & $\mathrm{H}$ & 0.28499 & -2.53823 & -1.38712 \\
\hline $\begin{array}{l}c \\
\mathrm{O}\end{array}$ & $\begin{array}{l}-7.70731 \\
-6.54176\end{array}$ & 2.92896 & 0.21044 & $\mathrm{H}$ & 2.48799 & -2.85315 & -0.29201 \\
\hline $\mathrm{C}$ & $\begin{array}{l}-6.54176 \\
-6.66674\end{array}$ & $\begin{array}{l}2.10607 \\
0.00650\end{array}$ & 0.17244 & $\mathrm{H}$ & 2.54274 & 3.10939 & 0.20271 \\
\hline $\mathrm{O}$ & -7.71391 & $\begin{array}{l}0.90059 \\
0.52905\end{array}$ & $\begin{array}{l}-0.42850 \\
-0.91757\end{array}$ & $\mathrm{H}$ & 0.74366 & 1.69810 & -0.68168 \\
\hline $\mathrm{C}$ & -5.40602 & 0.12311 & $\begin{array}{l}-0.91 / 5 / \\
-0.42156\end{array}$ & $\mathrm{H}$ & 0.38718 & 2.20955 & 0.97265 \\
\hline $\mathrm{C}$ & -5.40133 & -1.15023 & -1.02243 & $\mathrm{H}$ & 3.51227 & -1.43112 & -1.78109 \\
\hline $\mathrm{C}$ & -4.24361 & -1.91435 & $\begin{array}{l}-1.0 \angle 243 \\
-1.03546\end{array}$ & $\mathrm{H}$ & 6.05091 & -0.94840 & -2.16673 \\
\hline $\mathrm{C}$ & -3.06257 & -1.42237 & -0.44566 & $\mathrm{H}$ & 4.84033 & 0.24550 & -2.61901 \\
\hline $\mathrm{C}$ & -3.04036 & -0.14320 & $\begin{array}{r}-0.44500 \\
0.15154\end{array}$ & $\mathrm{H}$ & 6.40183 & 0.29849 & 0.02366 \\
\hline $\mathrm{C}$ & -4.21957 & 0.60697 & 0.15154 & $\mathrm{H}$ & 6.78591 & 1.28770 & -1.37396 \\
\hline $\mathrm{O}$ & -1.97266 & -2.21659 & & $\mathrm{H}$ & -0.28802 & -2.45019 & 2.39836 \\
\hline $\mathrm{C}$ & -0.81683 & -1.91731 & $\begin{array}{c}-0.508 / 1 \\
0.33588\end{array}$ & $\mathrm{H}$ & -1.95778 & -1.87166 & 2.21844 \\
\hline
\end{tabular}




$\begin{array}{rrrr}\mathrm{H} & -1.50973 & -3.49005 & 1.63389 \\ \mathrm{H} & 1.72364 & 0.64763 & 2.71215 \\ \mathrm{H} & 0.01031 & 0.25979 & 2.78590 \\ \mathrm{H} & 1.21094 & -1.03640 & 2.65929 \\ \mathrm{H} & 5.84238 & 3.51483 & -1.50705 \\ \mathrm{H} & 4.66491 & 2.57983 & -2.45999 \\ \mathrm{H} & 4.10057 & 3.73229 & -1.23056 \\ \mathrm{H} & 6.44768 & 2.85285 & 0.80734 \\ \mathrm{H} & 4.80252 & 3.36388 & 1.22408 \\ \mathrm{H} & 5.40671 & 1.78026 & 1.76344 \\ \mathrm{H} & 5.13322 & -0.48765 & 1.48286 \\ \mathrm{H} & 3.65583 & 0.09210 & 2.24507 \\ \mathrm{H} & 3.81426 & -1.61476 & 1.79848 \\ \mathrm{H} & 5.59045 & -2.12363 & -0.01055\end{array}$

Table S10. Cartesian Coordinates For The Re-Optimized Conformer 9 of

\section{Compound 1}

1a-opt_freq-conf-9.log

Energy: $-872859.0235437 \mathrm{kcal} / \mathrm{mol}$

$\begin{array}{lccc}\text { Atom } & \mathrm{X}(\AA) & \mathrm{Y} & \mathrm{Z} \\ \mathrm{C} & -8.81128 & 1.56161 & -0.65418 \\ \mathrm{O} & -7.62982 & 0.76327 & -0.71962 \\ \mathrm{C} & -6.53394 & 1.25400 & -0.10919 \\ \mathrm{O} & -6.53545 & 2.32122 & 0.47346 \\ \mathrm{C} & -5.35666 & 0.35814 & -0.23278 \\ \mathrm{C} & -5.40562 & -0.87326 & -0.91464 \\ \mathrm{C} & -4.27022 & -1.66807 & -0.99794 \\ \mathrm{C} & -3.06354 & -1.25125 & -0.40335 \\ \mathrm{C} & -2.98901 & -0.01473 & 0.27431 \\ \mathrm{C} & -4.14478 & 0.76553 & 0.34650 \\ \mathrm{O} & -1.99985 & -2.07185 & -0.54157 \\ \mathrm{C} & -0.81591 & -1.85862 & 0.28885 \\ \mathrm{C} & -0.49702 & -0.33932 & 0.27577 \\ \mathrm{C} & -1.67625 & 0.44475 & 0.87000 \\ \mathrm{C} & 0.29525 & -2.63103 & -0.43004 \\ \mathrm{C} & 1.69749 & -2.26591 & 0.06805 \\ \mathrm{C} & 1.96216 & -0.75356 & -0.05623 \\ \mathrm{C} & 0.92928 & 0.06076 & 0.80335 \\ \mathrm{C} & 3.47884 & -0.33394 & 0.04348 \\ \mathrm{C} & 3.55024 & 1.20451 & -0.26081 \\ \mathrm{C} & 2.60458 & 2.00993 & 0.64184\end{array}$

\begin{tabular}{|c|c|c|c|}
\hline $\mathrm{C}$ & 1.14697 & 1.56498 & 0.50329 \\
\hline $\mathrm{C}$ & 4.25912 & -1.10035 & -1.11251 \\
\hline $\mathrm{C}$ & 5.25955 & -0.21360 & -1.86710 \\
\hline $\mathrm{C}$ & 5.97243 & 0.76738 & -0.94342 \\
\hline $\mathrm{C}$ & 4.99394 & 1.82797 & -0.36883 \\
\hline $\mathrm{C}$ & -1.13612 & -2.47685 & 1.65638 \\
\hline $\mathrm{H}$ & -0.47540 & -0.08902 & -0.80044 \\
\hline $\mathrm{H}$ & 1.83455 & -2.60859 & 1.10670 \\
\hline $\mathrm{C}$ & 1.06301 & -0.15771 & 2.32990 \\
\hline $\mathrm{H}$ & 2.92295 & 1.94210 & 1.69412 \\
\hline $\mathrm{H}$ & 3.12674 & 1.29476 & -1.27772 \\
\hline $\mathrm{C}$ & 4.92839 & 3.01568 & -1.35496 \\
\hline $\mathrm{C}$ & 5.55031 & 2.35652 & 0.96828 \\
\hline $\mathrm{C}$ & 4.10281 & -0.69633 & 1.40930 \\
\hline $\mathrm{O}$ & 5.01034 & -2.21829 & -0.64918 \\
\hline $\mathrm{H}$ & 1.69696 & -0.49633 & -1.09905 \\
\hline $\mathrm{H}$ & -9.58604 & 1.00738 & -1.19814 \\
\hline $\mathrm{H}$ & -9.12081 & 1.72010 & 0.39018 \\
\hline $\mathrm{H}$ & -8.64762 & 2.54387 & -1.12325 \\
\hline $\mathrm{H}$ & -6.33872 & -1.20255 & -1.37489 \\
\hline $\mathrm{H}$ & -4.28706 & -2.62849 & -1.51912 \\
\hline $\mathrm{H}$ & -4.11113 & 1.73055 & 0.85981 \\
\hline $\mathrm{H}$ & -1.55828 & 1.52129 & 0.67674 \\
\hline $\mathrm{H}$ & -1.71204 & 0.33839 & 1.96814 \\
\hline $\mathrm{H}$ & 0.11204 & -3.71391 & -0.33347 \\
\hline $\mathrm{H}$ & 0.21956 & -2.38984 & -1.50292 \\
\hline $\mathrm{H}$ & 2.41956 & -2.83889 & -0.53506 \\
\hline $\mathrm{H}$ & 2.66806 & 3.07976 & 0.38525 \\
\hline $\mathrm{H}$ & 0.81083 & 1.77380 & -0.52831 \\
\hline $\mathrm{H}$ & 0.51496 & 2.17776 & 1.16711 \\
\hline $\mathrm{H}$ & 3.51036 & -1.44796 & -1.84941 \\
\hline $\mathrm{H}$ & 5.97576 & -0.88621 & -2.36482 \\
\hline $\mathrm{H}$ & 4.73892 & 0.33478 & -2.66873 \\
\hline $\mathrm{H}$ & 6.44857 & 0.20158 & -0.12645 \\
\hline $\mathrm{H}$ & 6.79361 & 1.27714 & -1.47519 \\
\hline $\mathrm{H}$ & -0.24693 & -2.55024 & 2.29413 \\
\hline $\mathrm{H}$ & -1.90015 & -1.90700 & 2.20448 \\
\hline $\mathrm{H}$ & -1.52391 & -3.49431 & 1.49705 \\
\hline $\mathrm{H}$ & 1.85461 & 0.46994 & 2.75766 \\
\hline $\mathrm{H}$ & 0.13424 & 0.11812 & 2.85055 \\
\hline $\mathrm{H}$ & 1.29854 & -1.19359 & 2.60520 \\
\hline $\mathrm{H}$ & 5.90350 & 3.52646 & -1.41351 \\
\hline $\mathrm{H}$ & 4.66344 & 2.68280 & -2.37188 \\
\hline $\mathrm{H}$ & 4.18139 & 3.76430 & -1.04628 \\
\hline $\mathrm{H}$ & 6.58557 & 2.70876 & 0.82449 \\
\hline $\mathrm{H}$ & 4.96906 & 3.20917 & 1.35207 \\
\hline
\end{tabular}




$\begin{array}{rrrr}\mathrm{H} & 5.57301 & 1.58285 & 1.74869 \\ \mathrm{H} & 5.19840 & -0.67523 & 1.37000 \\ \mathrm{H} & 3.78190 & -0.01313 & 2.20433 \\ \mathrm{H} & 3.82388 & -1.71162 & 1.72766 \\ \mathrm{H} & 4.42120 & -2.85689 & -0.22040\end{array}$

Table S11. Cartesian Coordinates For The Re-Optimized Conformer 10 of Compound 1

\begin{tabular}{lccc} 
1a-opt freq-conf-10.log & Energy: - \\
872859.0062432 kcal/mol & \\
\multicolumn{4}{c}{} \\
Atom & $\mathrm{X}(\AA)$ & $\mathrm{Y}$ & $\mathrm{Z}$ \\
$\mathrm{C}$ & -7.69654 & 2.93699 & 0.22767 \\
$\mathrm{O}$ & -6.53416 & 2.10968 & 0.18754 \\
$\mathrm{C}$ & -6.66188 & 0.91529 & -0.42269 \\
$\mathrm{O}$ & -7.70892 & 0.54507 & -0.91744 \\
$\mathrm{C}$ & -5.40372 & 0.12741 & -0.41875 \\
$\mathrm{C}$ & -5.40139 & -1.14011 & -1.03172 \\
$\mathrm{C}$ & -4.24565 & -1.90716 & -1.05021 \\
$\mathrm{C}$ & -3.06461 & -1.42364 & -0.45386 \\
$\mathrm{C}$ & -3.04017 & -0.15108 & 0.15671 \\
$\mathrm{C}$ & -4.21735 & 0.60235 & 0.16295 \\
$\mathrm{O}$ & -1.97585 & -2.21956 & -0.52465 \\
$\mathrm{C}$ & -0.81852 & -1.92923 & 0.31931 \\
$\mathrm{C}$ & -0.54043 & -0.40426 & 0.23231 \\
$\mathrm{C}$ & -1.75468 & 0.37648 & 0.75606 \\
$\mathrm{C}$ & 0.33006 & -2.70674 & -0.33195 \\
$\mathrm{C}$ & 1.70926 & -2.27659 & 0.17823 \\
$\mathrm{C}$ & 1.93599 & -0.76615 & -0.02022 \\
$\mathrm{C}$ & 0.86133 & 0.06285 & 0.77121 \\
$\mathrm{C}$ & 3.43805 & -0.30085 & 0.09158 \\
$\mathrm{C}$ & 3.47706 & 1.22148 & -0.28992 \\
$\mathrm{C}$ & 2.48845 & 2.04616 & 0.54698 \\
$\mathrm{C}$ & 1.04676 & 1.55483 & 0.39775 \\
$\mathrm{C}$ & 4.26492 & -1.10369 & -1.00584 \\
$\mathrm{C}$ & 5.26005 & -0.22984 & -1.78217 \\
$\mathrm{C}$ & 5.92533 & 0.81538 & -0.89423 \\
$\mathrm{C}$ & 4.90662 & 1.87712 & -0.39605 \\
$\mathrm{C}$ & -1.15462 & -2.48240 & 1.71056 \\
$\mathrm{H}$ & -0.49874 & -0.21062 & -0.85492 \\
$\mathrm{H}$ & 1.82953 & -2.56056 & 1.23644 \\
$\mathrm{C}$ & 0.96366 & -0.07238 & 2.30986 \\
$\mathrm{H}$ & 2.78222 & 2.04144 & 1.60857
\end{tabular}

$\begin{array}{lrrr}\mathrm{H} & 3.07693 & 1.24825 & -1.31989 \\ \mathrm{C} & 4.83463 & 3.01210 & -1.44204 \\ \mathrm{C} & 5.41725 & 2.48607 & 0.92497 \\ \mathrm{C} & 4.03811 & -0.57727 & 1.48762 \\ \mathrm{O} & 5.03302 & -2.17630 & -0.46894 \\ \mathrm{H} & 1.68936 & -0.57138 & -1.08095 \\ \mathrm{H} & -7.40267 & 3.85279 & 0.75521 \\ \mathrm{H} & -8.04137 & 3.17923 & -0.78921 \\ \mathrm{H} & -8.51649 & 2.43638 & 0.76497 \\ \mathrm{H} & -6.32167 & -1.51010 & -1.48918 \\ \mathrm{H} & -4.22468 & -2.89426 & -1.51870 \\ \mathrm{H} & -4.20898 & 1.59204 & 0.62562 \\ \mathrm{H} & -1.66100 & 1.44406 & 0.50753 \\ \mathrm{H} & -1.81397 & 0.32806 & 1.85731 \\ \mathrm{H} & 0.17373 & -3.78780 & -0.18230 \\ \mathrm{H} & 0.27409 & -2.52427 & -1.41755 \\ \mathrm{H} & 2.46098 & -2.86002 & -0.37667 \\ \mathrm{H} & 2.53025 & 3.10267 & 0.23650 \\ \mathrm{H} & 0.73098 & 1.70076 & -0.65096 \\ \mathrm{H} & 0.38273 & 2.18362 & 1.01363 \\ \mathrm{H} & 3.54298 & -1.50795 & -1.74059 \\ \mathrm{H} & 6.00495 & -0.90732 & -2.22847 \\ \mathrm{H} & 4.74497 & 0.26320 & -2.62239 \\ \mathrm{H} & 6.39666 & 0.30456 & -0.03910 \\ \mathrm{H} & 6.74529 & 1.31961 & -1.43308 \\ \mathrm{H} & -0.28007 & -2.49299 & 2.37211 \\ \mathrm{H} & -1.94892 & -1.90752 & 2.20804 \\ \mathrm{H} & -1.50793 & -3.51853 & 1.59830 \\ \mathrm{H} & 1.72644 & 0.59969 & 2.72222 \\ \mathrm{H} & 0.01491 & 0.20221 & 2.79390 \\ \mathrm{H} & 1.22179 & -1.08519 & 2.64424 \\ \mathrm{H} & 5.79776 & 3.54466 & -1.50450 \\ \mathrm{H} & 4.60218 & 2.62211 & -2.44654 \\ \mathrm{H} & 4.06212 & 3.75538 & -1.18826 \\ \mathrm{H} & 6.44583 & 2.85941 & 0.78658 \\ \mathrm{H} & 5.40471 & 3.34010 & 1.25301 \\ \mathrm{H} & 3.13348 & -0.53009 & 1.47200 \\ \mathrm{H} & 3.77680 & -1.58200 & 1.85100 \\ \mathrm{H} & & -2.80904 & -0.02330\end{array}$


Figure S15. Comparison of Calculated ECD spectra for 1 and Similarity Factors

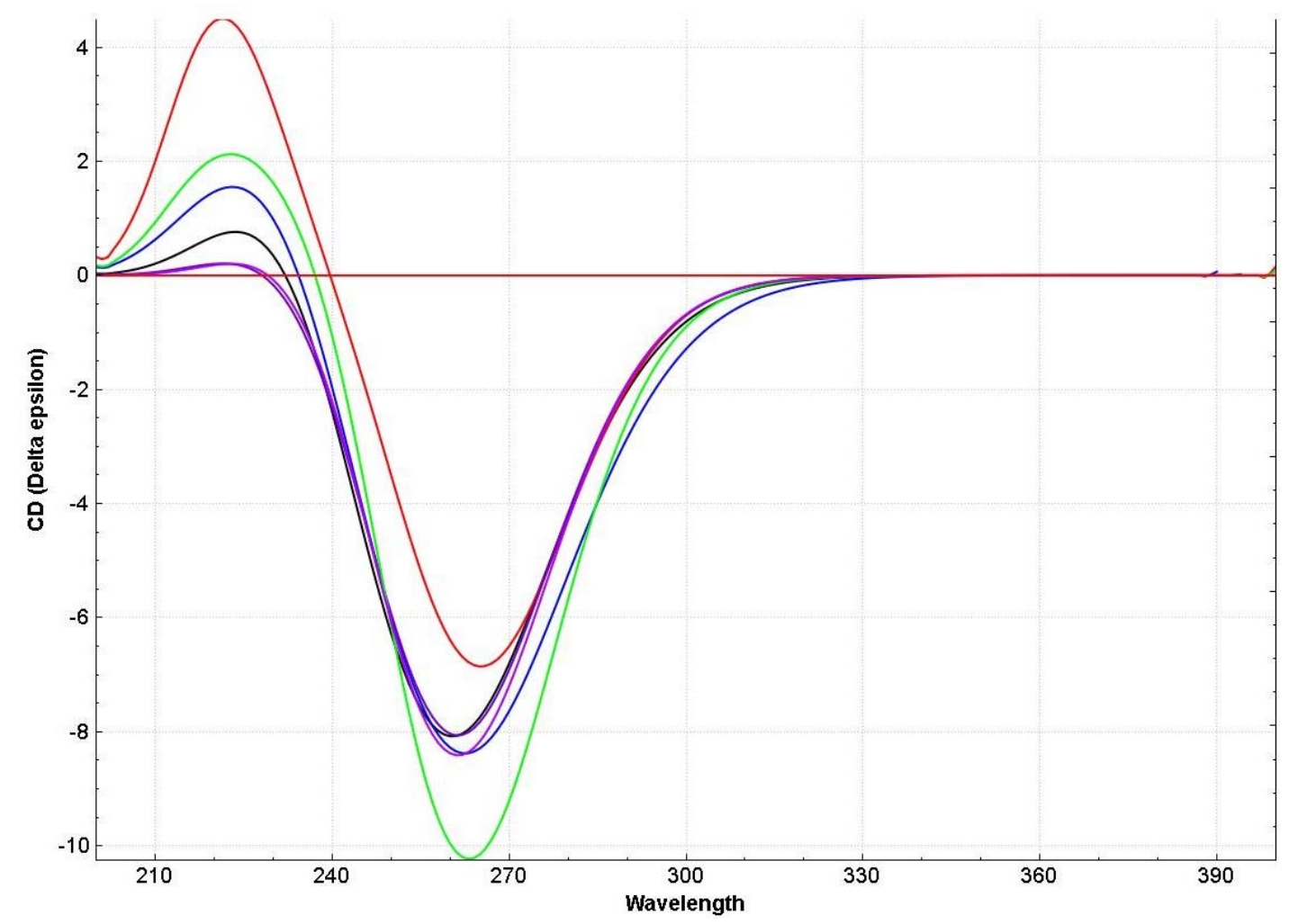

\begin{tabular}{|l|l|l|l|l|l|}
\hline Color & Level & $\begin{array}{l}\text { Shift } \\
(\mathbf{n m})\end{array}$ & $\Delta_{\text {ESI }}{ }^{1}$ & \multicolumn{2}{|c|}{ similarity factors $^{1}$} \\
\hline & & & & $1 R, 5 S, 8 R, 9 S, 13 S, 14 S$ & Enantiomer \\
\hline Black & B3LYP/DEF2SVP & -5 & 0.9135 & 0.9232 & 0.0097 \\
\hline Blue & B3LYP/DEF2TZVPP & -10 & 0.9113 & 0.9341 & 0.0228 \\
\hline Red & BH\&HLYP/DEF2TZVPP & +17 & 0.875 & 0.9077 & 0.0324 \\
\hline Purple & CAM-B3LYP/DEF2TZVPP & +7 & 0.9241 & 0.9351 & 0.0110 \\
\hline Green & M062X/DEF2TZVPP & +13 & 0.8845 & 0.9256 & 0.0411 \\
\hline Pink & Wb97xd/DEF2TZVPP & +9 & 0.9184 & 0.9254 & 0.0070 \\
\hline
\end{tabular}

(1) Bruhn, T.; Schaumloffel, A.; Hemberger, Y.; Bringmann, G. Chirality 2013, 25, 243-249. 